\title{
Prototypic and Arkypallidal Neurons in the Dopamine-Intact External Globus Pallidus
}

\author{
Azzedine Abdi, ${ }^{1,2 *}$ (icolas Mallet, ${ }^{1,2,3 *}$ Foad Y. Mohamed, ${ }^{3}$ Andrew Sharott, ${ }^{3}$ @Paul D. Dodson, ${ }^{3,4}$ \\ ĐKouichi C. Nakamura, ${ }^{3}$ Sana Suri, ${ }^{3}$ Sophie V. Avery, ${ }^{3}{ }^{-}$Joseph T. Larvin, ${ }^{3}$ Farid N. Garas, ${ }^{3}$ Shady N. Garas, ${ }^{3}$

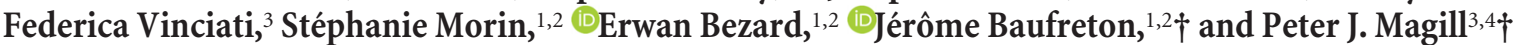 \\ ${ }^{1}$ Université de Bordeaux, Institut des Maladies Neurodégénératives, F-33000 Bordeaux, France, ${ }^{2}$ Centre National de la Recherche Scientifique Unité Mixte \\ de Recherche 5293, Institut des Maladies Neurodégénératives, F-33000 Bordeaux, France, ${ }^{3}$ Medical Research Council Brain Network Dynamics Unit, \\ Department of Pharmacology, University of Oxford, Oxford OX1 3QT, United Kingdom, and ${ }^{4}$ Oxford Parkinson's Disease Centre, University of Oxford, \\ Oxford OX1 3QX, United Kingdom
}

Studies in dopamine-depleted rats indicate that the external globus pallidus (GPe) contains two main types of GABAergic projection cell; so-called "prototypic" and "arkypallidal" neurons. Here, we used correlative anatomical and electrophysiological approaches in rats to determine whether and how this dichotomous organization applies to the dopamine-intact GPe. Prototypic neurons coexpressed the transcription factors Nkx2-1 and Lhx6, comprised approximately two-thirds of all GPe neurons, and were the major GPe cell type innervating the subthalamic nucleus (STN). In contrast, arkypallidal neurons expressed the transcription factor FoxP2, constituted just over one-fourth of GPe neurons, and innervated the striatum but not STN. In anesthetized dopamine-intact rats, molecularly identified prototypic neurons fired at relatively high rates and with high regularity, regardless of brain state (slow-wave activity or spontaneous activation). On average, arkypallidal neurons fired at lower rates and regularities than prototypic neurons, and the two cell types could be further distinguished by the temporal coupling of their firing to ongoing cortical oscillations. Complementing the activity differences observed in vivo, the autonomous firing of identified arkypallidal neurons in vitro was slower and more variable than that of prototypic neurons, which tallied with arkypallidal neurons displaying lower amplitudes of a "persistent" sodium current important for such pacemaking. Arkypallidal neurons also exhibited weaker driven and rebound firing compared with prototypic neurons. In conclusion, our data support the concept that a dichotomous functional organization, as actioned by arkypallidal and prototypic neurons with specialized molecular, structural, and physiological properties, is fundamental to the operations of the dopamine-intact GPe.

Key words: anatomy; arkypallidal; basal ganglia; electrophysiology; globus pallidus; transcription factor

\section{Introduction}

Most circuit-level schemes of basal ganglia (BG) organization embody the external globus pallidus (GPe) as a homogeneous

Received Nov. 11, 2014; revised Feb. 16, 2015; accepted Feb. 23, 2015.

Author contributions: N.M., K.C.N., J.B., and P.J.M. designed research; A.A., N.M., F.Y.M., A.S., P.D.D., K.C.N., S.S., S.V.A., J.T.L., F.N.G., S.N.G., F.V., S.M., J.B., and P.J.M. performed research; E.B. contributed unpublished reagents/ analytic tools; A.A., N.M., F.Y.M., A.S., P.D.D., K.C.N., S.S., S.V.A., J.T.L., F.N.G., S.N.G., S.M., J.B., and P.J.M. analyzed data; J.B. and P.J.M. wrote the paper.

This work was supported by the Medical Research Council United Kingdom Awards UU138197109 and MC_UU_12020/5, Parkinson's UK Grant G-0806 and Monument Trust Discovery Award J-0901, Wellcome Trust Investigator Award 101821 to P.J.M., Association France Parkinson Ph.D. fellowship to A.A., Fondation de France, and Agence Nationale de la Recherche Grant 08-JCIC-0087 and Grant LABEX BRAIN ANR-10-LABX-43. A.S. was supported in part by the European Union Marie Curie European Re-integration Grant SNAP-PD. K.C.N. was supported in part by the Human Frontier Science Program (LT000396/2009-L). S.V.A. was supported in part by a Wellcome Trust Doctoral Studentship in Neuroscience 099711. F.N.G. was supported in part by a University of Oxford Clarendon Fund Scholarship. We thank S. Arber, A. Buchberg, C. Cozzari, and S. McKnight for gifts of antibodies; S. Butt, N. Doig, J. Kaufling, L. Magno, V. Pachnis, and N. Kessaris for insightful scientific discussions; the Bordeaux Imaging Center of the University of Bordeaux Segalen; and E. Norman, B. Micklem, L. Conyers, C. Johnson, C. Poujol, and S. Marais for technical assistance.

The authors declare no competing financial interests.

*A.A. and N.M. contributed equally to this work.

†J.B. and P.J.M. contributed equally to this work as co-senior authors.

This article is freely available online through the J Neurosci Author Open Choice option. entity (Albin et al., 1989; Mink, 1996; Wichmann and DeLong, 1996; Redgrave et al., 1999; Nambu et al., 2002). For example, the influential "direct/indirect pathways" model (DeLong, 1990; Smith et al., 1998) posits that GPe is populated by a single GABAergic cell type that is conceptually "downstream" of striatum and "upstream" of the subthalamic nucleus (STN). Although such schemes are necessarily idealized, this notion of functional homogeneity is nevertheless contradicted by a host of evidence of cellular diversity and functional specialization within GPe. Indeed, electrophysiological diversity has long been observed in GPe, with some of the first recordings in awake primates distinguishing two major groups of GPe neurons based on their firing rates and patterns (DeLong, 1971). Moreover, it was evi-

Correspondence should be addressed to either of the following: Dr. Peter J. Magill, Medical Research Council Brain Network Dynamics Unit, Department of Pharmacology, University of Oxford, Mansfield Road, Oxford 0X1 30T, United Kingdom, E-mail: peter.magill@pharm.ox.ac.uk; or Dr Jérôme Baufreton, Institut des Maladies Neurodégénératives, Unité Mixte de Recherche Centre National de la Recherche Scientifique 5293, 146 Rue Léo Saignat, 33076 Bordeaux Cedex, France. E-mail: jerome.baufreton@u-bordeaux2.fr.

D0I:10.1523/JNEUROSCI.4662-14.2015

Copyright $\odot 2015$ Abdi et al.

This is an Open Access article distributed under the terms of the Creative Commons Attribution License Creative Commons Attribution 4.0 International, which permits unrestricted use, distribution and reproduction in any medium provided that the original work is properly attributed. 
dent from early studies of rodent pallidum that GPe neurons have diverse intrinsic membrane properties (Kita and Kitai, 1991; Nambu and Llinaś, 1994). The molecular expression profiles of GPe neurons also vary, which partly stems from their diverse developmental origins (Kita, 2007; Flandin et al., 2010; NóbregaPereira et al., 2010). Neurons in GPe likewise exhibit diverse somatodendritic and axonal structure (Kita, 2007); although most neurons innervate STN, some also target one or more or all of the other BG nuclei (Bevan et al., 1998; Smith et al., 1998; Sato et al., 2000). It is thus apparent that defining the nature and necessity of GPe neuron heterogeneity is imperative for understanding BG function and dysfunction.

To progress toward comprehensive definitions of GPe cell types, diverse and highly dynamic electrophysiological phenotypes require framing in the context of neuronal properties that are fixed over longer timescales, such as molecular profiles and structure. Studies in dopamine-depleted parkinsonian rats suggest that GPe has a dichotomous functional organization that arises from the existence of two major GABAergic projection cell types, "prototypic" neurons and "arkypallidal” neurons (Mallet et al., 2012). Prototypic GPe neurons conform to conventional views of GPe cell functions because they fire tonically at high rates in vivo, often express parvalbumin (PV), and always innervate STN. In contrast, arkypallidal neurons fire differently, express preproenkephalin (PPE), and innervate only striatum (Mallet et al., 2012). Therefore, in the dopamine-depleted state at least, two populations of GPe neuron can orchestrate activities across the entire BG in a cell-type-specific manner. However, it is unknown whether and to what extent the core cellular determinants of this dichotomous GPe organization extend to the healthy, dopamine-intact brain.

To address these key issues, we used stereological analyses of immunofluorescently labeled neurons in dopamine-intact GPe to elucidate the definitive molecular architecture of prototypic and arkypallidal neurons, and to generate unbiased estimates of their population sizes. Exploiting the unambiguous molecular signatures of these two cell types, we then defined the brain statedependent firing of identified neurons in vivo as well as the autonomous firing and intrinsic membrane properties of identified neurons in vitro. Our data provide unique insights into how these dichotomous cell types could subserve a division of labor in GPe.

\section{Materials and Methods}

Experimental procedures were performed on male Sprague Dawley rats (Charles River) and were conducted either in Oxford in accordance with the Animals (Scientific Procedures) Act, 1986 (United Kingdom), or in Bordeaux according to institutional guidelines and the European Communities Council Directive 86/609/EEC and its successor 2010/63/EU. All experimental work adhered to Society for Neuroscience Policies on the Use of Animals in Neuroscience Research.

Quantification of molecular marker expression in populations of GPe neurons. Fifteen adult rats (3-4 months old; 290-430 g) and 20 juvenile rats (postnatal days $[\mathrm{P}] 28-35)$ were killed with pentobarbital $(1.5 \mathrm{~g} / \mathrm{kg}$, i.p.; Ayrton Saunders) and transcardially perfused with $0.05 \mathrm{~m} \mathrm{PBS,} \mathrm{pH}$ 7.4 (PBS), followed by $4 \% \mathrm{w} / \mathrm{v}$ PFA in $0.1 \mathrm{~m}$ phosphate buffer, $\mathrm{pH} 7.4$ (PB). Adult rat brains were left overnight in fixative at $4^{\circ} \mathrm{C}$ and then stored in PBS at $4^{\circ} \mathrm{C}$ before being cut into $50 \mu \mathrm{m}$ coronal sections on a vibrating microtome (VT1000S; Leica Microsystems). Juvenile rat brains were left overnight in fixative at $4^{\circ} \mathrm{C}$, immersed in PBS containing $20 \%$ $\mathrm{w} / \mathrm{v}$ sucrose for $24 \mathrm{~h}$, and then stored in this solution at $-80^{\circ} \mathrm{C}$ before being cut into $50 \mu \mathrm{m}$ coronal sections on a cryostat (CM3000; Leica Microsystems). Free-floating tissue sections were collected in series, washed in PBS, and those containing "rostral," "central," and "caudal" GPe were selected (see Fig. 1A; in adults, these sections, respectively, correspond to approximate distances of $0.6,1.0$, and $1.4 \mathrm{~mm}$ posterior to Bregma) (Paxinos and Watson, 2007) and then processed for indirect immunofluorescence to reveal molecular markers (Mallet et al., 2012; Sharott et al., 2012; Nakamura et al., 2014). Briefly, after 1-2 h of incubation in "Triton PBS" (PBS with $0.3 \% \mathrm{v} / \mathrm{v}$ Triton X-100 and $0.02 \% \mathrm{w} / \mathrm{v}$ sodium azide; Sigma) containing 10\% v/v normal donkey serum (Jackson ImmunoResearch Laboratories), sections were incubated overnight at room temperature in Triton PBS containing 1\% v/v normal donkey serum and a mixture of between two and four of the following primary antibodies: goat anti-choline acetyltransferase (ChAT; 1:500 dilution; Millipore, AB144P) (Stacy et al., 2005); mouse anti-ChAT (1:1000; gift from C. Cozzari) (von Engelhardt et al., 2007); rabbit anti-ER81 (also known as ETS variant gene 1 protein; 1:5000; gift from S. Arber) (Arber et al., 2000); goat anti-forkhead box protein P2 (FoxP2; 1:500; Santa Cruz Biotechnology, sc-21069); rabbit anti-FoxP2 (1:1000; Sigma, HPA000382) (Reimers-Kipping et al., 2011); mouse anti-human neuronal protein $\mathrm{HuC} / \mathrm{HuD}$ ( $\mathrm{HuCD}$; 1:200; Invitrogen, A-21271, clone 16A11) that specifically binds to an epitope present in gene products of several $\mathrm{Hu}$ genes, most notably of $\mathrm{HuC}$ and $\mathrm{HuD}$ (Marusich et al., 1994; Pascale et al., 2004); mouse anti-LIM homeobox protein 6 (Lhx6; 1:2000; Santa Cruz Biotechnology, sc-271433); this monoclonal antibody recognizes a single protein band of the predicted molecular mass of Lhx6 on Western blots of cell lysates (manufacturer's data); rabbit anti-meis homeobox 2 protein (Meis2; 1:2000; gift from A. Buchberg) (Swift et al., 1998; Agoston and Schulte, 2009); mouse anti-neuronal nuclei protein (NeuN, also known as hexaribonucleotide-binding protein 3; 1:500; Millipore, MAB377, clone A60); rabbit anti-neuronal PAS domain protein 1 (Npas1; 1:500; gift from S.L. McKnight) (Erbel-Sieler et al., 2004); rabbit anti-NK2 homeobox 1 protein (Nkx2-1, also known as thyroid transcription factor 1; 1:500; Santa Cruz Biotechnology, sc-13040) (Magno et al., 2011), or mouse anti-Nkx2-1 (1:50; Leica Biosystems, NCL-TTF-1), which were raised against different $\mathrm{Nkx} 2-1$ peptide antigens but resulted in a $99.5 \%$ overlap in immunoreactive neurons; guinea pig anti-PV (1: 1000; Synaptic Systems, 195004) (Massi et al., 2012); and rabbit anti-PPE (1:5000; LifeSpan Biosciences, LS-C23084); in control experiments, the use of this PPE antiserum resulted in immunolabeling that was indistinguishable from that achieved with an affinity-purified antibody raised against PPE in guinea pigs (Lee et al., 1997). To optimize immunolabeling of Meis2, Npas1, and PPE, we used a heat pretreatment $(2,2$, and $6 \mathrm{~h}$, respectively) as a means of antigen retrieval (Mallet et al., 2012). After exposure to primary antibodies, sections were washed in PBS and incubated overnight at room temperature in Triton PBS containing a mixture of secondary antibodies (all raised in donkey) that were conjugated to the following fluorophores: AMCA (1:250 dilution; Jackson ImmunoResearch Laboratories); AlexaFluor-488 (1:500; Invitrogen); Cy3 (1: 1000; Jackson ImmunoResearch Laboratories); or DyLight 649 (1: 500; Jackson ImmunoResearch Laboratories). All secondary antibodies were highly cross-adsorbed by the manufacturers to reduce cross-species reactivity. After washing in PBS, sections were mounted in Vectashield (Vector Laboratories) and imaged on an epifluorescence microscope (Carl Zeiss, AxioImager.M2) running Axiovision software (Carl Zeiss) and equipped with a StereoInvestigator system (MBF Bioscience). Appropriate sets of filter cubes were used to image the fluorescence channels: AMCA (excitation 299-392 nm, beamsplitter $395 \mathrm{~nm}$, emission 420-470 nm); AlexaFluor-488 (excitation 450-490 nm, beamsplitter $495 \mathrm{~nm}$, emission 500-550 nm); Cy3 (excitation 532-558 nm, beamsplitter $570 \mathrm{~nm}$, emission 570-640 nm); and DyLight 649 (excitation $625-655 \mathrm{~nm}$, beamsplitter $660 \mathrm{~nm}$, emission 665-715 nm). Images of each of the channels were taken sequentially and separately to negate possible crosstalk of signal across channels.

Using a series of partly overlapping, complementary immunofluorescence protocols, GPe neurons were tested for their combinatorial expression of all the molecular markers described above. ROIs (i.e., the borders of rostral, central, and caudal GPe) were first defined using a $5 \times 0.16 \mathrm{NA}$ objective lens. The medial and lateral borders of GPe were readily delineated according to the internal capsule and dorsal striatum, respectively (see Fig. 1A). The ventral border of GPe was conservatively assigned such that it was set well apart from the poorly defined boundary between GPe and the functionally distinct ventral pallidum (see Fig. $1 A$, red lines). For the purposes of this study, it was not necessary to determine absolute numbers or densities of GPe neurons expressing these markers. We thus 
used a version of design-based stereology, the "modified optical fractionator" (West, 1999, 2012), to generate unbiased cell counts and map cell distributions and determine the proportions of a given population of neurons that express certain combinations of markers. Stereological sampling was performed over the entire GPe in both hemispheres, as sectioned at the designated rostral, central, and caudal levels. For each immunofluorescence protocol, a series of completely tessellated, $z$-stacked images were acquired using a $40 \times 1.3 \mathrm{NA}$ oil-immersion objective lens and $1.0 \mu \mathrm{m}$ steps ("optical sections") at depths of $2-12 \mu \mathrm{m}$ from the upper surface of each section at the level of GPe. To minimize confounds arising from surface irregularities, neuropil within a $2-\mu \mathrm{m}-$ thick "guard zone" at the upper surface was not imaged. This sampling strategy thus defined a $10-\mu \mathrm{m}$-thick "optical disector" that was used with abutting, unbiased $2 \mathrm{D}$ counting frames $(200 \times 150 \mu \mathrm{m}$; consisting of two perpendicular exclusion lines and two inclusion lines) to generate all cell counts and marker expression profiles (West, 1999, 2012; Glaser et al., 2007). A neuron was only counted once through the series of optical sections when its nucleus came into sharp focus within the disector; neurons with nuclei already in focus in the top optical section of the disector were ignored (West, 1999, 2012; Glaser et al., 2007). The use of stereology, and this optical disector probe in particular, ensured that we could generate robust and unbiased cell counts in a timely manner. For a given molecular marker, $\mathrm{X}$, we designate positive immunoreactivity (confirmed expression) as $\mathrm{X}^{+}$, and undetectable immunoreactivity (no expression) as $\mathrm{X}^{-}$. In this and related immunofluorescence analyses, a neuron was classified as not expressing the tested molecular marker only when positive immunoreactivity could be observed in other cells on the same optical section as the tested neuron. Immunoreactivity was scored (either positive or undetectable) by experienced investigators; the results of most immunofluorescence protocols were scored by at least two individuals, and the variability in the normalized scores of multiple investigators was low $(<5 \%)$. Each immunofluorescence protocol was repeated (and thus, each neuronal expression profile was quantified) at the three levels along the rostrocaudal axis of GPe in a minimum of three adult rats. Data from rostral, central, and caudal GPe of both hemispheres were exported to Excel (Microsoft) and, unless stated otherwise, were pooled for further analyses. In the bar graphs shown in figures, gray bars represent grand averages from all rats tested, with each superimposed black dot representing the average from an individual rat to give an indication of cohort variance.

Viral vector-mediated retrograde tract-tracing. To elucidate the axonal projections of populations of GPe neurons, we performed retrograde tracttracing using a recombinant, pseudotyped lentiviral vector (RV-G-MNDEGFP-WPRE) injected into either the STN or dorsal striatum. The RV-GMND-EGFP-WPRE vector is based on HIV Type 1, is pseudotyped with rabies virus glycoprotein, and contains a gene encoding enhanced green fluorescent protein (EGFP). Intracerebral injection of this vector results in the delivery of the EGFP transgene to neurons through retrograde transport from their axon terminal fields; indeed, its utility for retrogradely labeling several different cell types that provide synaptic inputs to striatum has been demonstrated in rodents (Kato et al., 2007). Eleven adult rats (290-310 g) were anesthetized with isoflurane ( $3 \%$ and $1.5 \% \mathrm{v} / \mathrm{v}$ in $\mathrm{O}_{2}$ for induction and maintenance, respectively), and then placed in a stereotaxic frame (Kopf). Wound margins were infiltrated with local anesthetic (xylocaine), and body temperature was maintained at $37 \pm 0.5^{\circ} \mathrm{C}$ by a homeothermic heating device (Harvard Apparatus). In five rats, discrete bilateral injections of RVG-MND-EGFP-WPRE vector $\left(0.2 \mu \mathrm{l}\right.$ per site, titer of $3.4 \times 10^{9}$ infectious particles $/ \mathrm{ml}$ ) were made into the STN $(3.8 \mathrm{~mm}$ posterior and $2.5 \mathrm{~mm}$ lateral of Bregma, $7.5 \mathrm{~mm}$ from brain surface) (Paxinos and Watson, 2007). In another six rats, discrete bilateral injections of the same vector $(0.4 \mu \mathrm{l}$ per site) were made into a central region of dorsal striatum $(1.8 \mathrm{~mm}$ anterior and $2.5 \mathrm{~mm}$ lateral of Bregma, $5.0 \mathrm{~mm}$ from brain surface). Allowing $17 \mathrm{~d}$ for neuronal transduction and labeling with EGFP, animals were killed and transcardially perfused with $100 \mathrm{ml}$ of PBS, followed by $300 \mathrm{ml}$ of $4 \% \mathrm{w} / \mathrm{v}$ PFA in PB. Brains were left overnight in fixative at $4^{\circ} \mathrm{C}$ and then stored for $3-7 \mathrm{~d}$ in $\mathrm{PBS}$ at $4^{\circ} \mathrm{C}$ before sectioning. Coronal sections $(50 \mu \mathrm{m})$ were cut from each brain using a vibrating microtome, collected in series, washed in PBS, and processed for indirect immunofluorescence using the antibodies and protocols described above. Transduced neurons were revealed after se- quential incubation with rat anti-green fluorescent protein (1:500; Nacalai Tesque, 04404-84) and donkey anti-rat conjugated to AlexaFluor-488 (1: 500; Invitrogen). The borders of the STN were readily delineated by immunoreactivity for FoxP2 (Campbell et al., 2009). To minimize the possibility of GPe neurons being transduced through their local axon collaterals during vector injections into striatum, we ensured that the outer limits of the striatal zones containing many $\mathrm{EGFP}^{+}$cells, which presumably indicated sites of vector injection, were $>400 \mu \mathrm{m}$ away from the rostral border of GPe. Neurons in the GPe-expressing EGFP were tested for coexpression of FoxP2, Nkx2-1, PV, and/or Npas1. After binding of primary and secondary antibodies, and final washing in PBS, sections were mounted in Vectashield and imaged on an epifluorescence microscope as described above, with the exception that GPe neurons expressing EGFP were imaged and counted throughout the whole depth of the tissue (excluding upper and lower guard zones). For the immunofluorescence images shown in Figures 123-4, digital images generated in this and preceding anatomical experiments were acquired as $z$-stacks (optical section thickness: $0.8-1.5 \mu \mathrm{m}$ ) using an epifluorescence or confocal microscope (Carl Zeiss LSM 710; see below for laser and filter settings). After selecting the thinnest $z$-stack necessary to convey the essential information (3-10 $\mu \mathrm{m}$ stack for Figs. 12-3; 18-28 $\mu \mathrm{m}$ for Fig. 4), raw images were cropped to ROIs, with brightness and contrast adjusted when necessary, using Photoshop software (Creative Suite 3; Adobe Systems).

In vivo electrophysiological recording and juxtacellular labeling of individual GPe neurons. Recording and labeling experiments were performed in 20 anesthetized dopamine-intact rats (3-4 months old, 305-400 g) and 16 anesthetized 6-hydroxydopamine (6-OHDA)-lesioned rats (3-5 months old and 295-470 g at the time of recording), as previously described (Mallet et al., 2008a, b, 2012). Briefly, anesthesia was induced with $4 \% \mathrm{v} / \mathrm{v}$ isoflurane in $\mathrm{O}_{2}$ and maintained with urethane $(1.3 \mathrm{~g} / \mathrm{kg}$, i.p.; ethyl carbamate, Sigma), and supplemental doses of ketamine $(30 \mathrm{mg} / \mathrm{kg}$, i.p.; Willows Francis) and xylazine (3 mg/kg, i.p.; Bayer). Wound margins were infiltrated with local anesthetic $(0.5 \% \mathrm{w} / \mathrm{v}$ bupivacaine; Astra). Animals were then placed in a stereotaxic frame (Kopf). Body temperature was maintained at $37 \pm 0.5^{\circ} \mathrm{C}$ by a homeothermic heating device (Harvard Apparatus). Electrocorticograms (ECoGs) and respiration rate were monitored constantly to ensure the animals' well-being. The epidural ECoG was recorded above the frontal (somatic sensory-motor) cortex ( $4.0 \mathrm{~mm}$ anterior and $2.0 \mathrm{~mm}$ lateral of bregma) (Paxinos and Watson, 2007) and was referenced against the ipsilateral cerebellar hemisphere (Mallet et al., 2012). Raw ECoG was bandpass filtered $(0.3-1500 \mathrm{~Hz},-3$ dB limits) and amplified (2000×; DPA-2FS filter/amplifier; NPI Electronic Instruments) before acquisition. Extracellular recordings of single-unit activity, that is, the action potentials ("spikes") fired by individual neurons in the GPe, were made using standard-wall borosilicate glass electrodes ( $10-30 \mathrm{M} \Omega$ in situ; tip diameter $\sim 1.2 \mu \mathrm{m}$ ) containing $0.5 \mathrm{M} \mathrm{NaCl}$ solution and neurobiotin $(1.5 \% \mathrm{w} / \mathrm{v}$; Vector $)$. Electrodes were lowered into the brain under stereotaxic guidance and using a computercontrolled stepper motor (IVM-1000; Scientifica), which allowed electrode placements to be made with submicron precision. Electrode signals were amplified $(10 \times)$ through the bridge circuitry of an Axoprobe-1A amplifier (Molecular Devices), AC-coupled, amplified another 100X, and filtered at $300-5000 \mathrm{~Hz}$ (DPA-2FS filter/amplifier). The ECoG and single-unit activity were each sampled at $17.9 \mathrm{kHz}$ using a Power1401 Analog-Digital converter and a PC running Spike2 acquisition and analysis software (Cambridge Electronic Design). As described previously (Mallet et al., 2008a, 2012), single-unit activity in GPe was recorded during cortical slow-wave activity (SWA), which is similar to activity observed during natural sleep, and/or during episodes of spontaneous "cortical activation," which contain patterns of activity that are more analogous to those observed during the awake, behaving state (Steriade, 2000). It is important to note that the neuronal activity patterns present under this anesthetic regimen may only be qualitatively similar to those present in the unanesthetized brain. Nevertheless, the urethaneanesthetized animal still serves as a useful model for assessing the impact of extremes of brain state on functional connectivity within and between the BG and cortex in dopamine-intact and parkinsonian animals (Magill et al., 2006; Mallet et al., 2008a, b; Sharott et al., 2012). Hindpaw withdrawal reflexes were not present during episodes of prolonged, sponta- 
neous cortical activation, thus indicating that anesthesia was adequate throughout recordings. Following electrophysiological recordings, single neurons were juxtacellularly labeled with neurobiotin (Magill et al., 2001; Sadek et al., 2007; Baufreton et al., 2009; Mallet et al., 2012). Briefly, positive current pulses ( $2-10 \mathrm{nA}, 200 \mathrm{~ms}, 50 \%$ duty cycle) were applied until the single-unit activity became robustly entrained by the pulses. Single-unit entrainment resulted in just one neuron being labeled with neurobiotin. Two to six hours after labeling, animals were killed and transcardially perfused with $100 \mathrm{ml}$ of PBS, followed by $300 \mathrm{ml}$ of $4 \% \mathrm{w} / \mathrm{v}$ $\mathrm{PFA}$ in PB. Brains were left overnight in fixative at $4^{\circ} \mathrm{C}$ and then stored for $1-3 \mathrm{~d}$ in PBS at $4^{\circ} \mathrm{C}$ before sectioning.

Molecular characterization of recorded and juxtacellularly labeled neurons. Parasagittal sections $(50 \mu \mathrm{m})$ were cut from each brain using a vibrating microtome, collected in series, and washed in PBS. Freefloating sections were then incubated overnight at room temperature in Triton PBS containing Cy3-conjugated streptavidin (1:1000 dilution; Invitrogen). Sections containing neurobiotin-labeled neuronal somata (those marked with $\mathrm{Cy} 3$ ) were then isolated for molecular characterization by indirect immunofluorescence using the antibodies and protocols described above. Recorded and identified GPe neurons were tested for expression of FoxP2, Nkx2-1, PPE, and/or PV. After binding of primary and secondary antibodies, and final washing in PBS, sections were mounted in Vectashield and imaged on confocal fluorescence microscopes (Carl Zeiss, LSM 510 or LSM 710), as previously described (Mallet et al., 2012; Sharott et al., 2012; Nakamura et al., 2014). Briefly, verification of molecular identity was performed by assessing single-plane confocal images acquired using a constant $1.0-\mu \mathrm{m}$-thick optical section (obtained by varying the pin hole size) and a $40 \times 1.3 \mathrm{NA}$ or a $63 \times 1.4 \mathrm{NA}$ oil-immersion objective lens. Each fluorescence channel was imaged in a separate track. Cy3 fluorescence signal was imaged with excitation from a helium/neon $543 \mathrm{~nm}$ laser, with emission restricted on the LSM 510 by a LP 560 filter (for the shorter wavelengths) and a NFT 635 VIS $2^{\circ}$ dichroic (for longer wavelengths). Emission on the LSM 710 was restricted by selecting an emission range of 552-639 $\mathrm{nm}$. AlexaFluor- 488 fluorescence was imaged with excitation from an argon $488 \mathrm{~nm}$ laser, with emission restricted by either a BP 510-530 filter (on the LSM 510) or by setting emission at 493-542 nm (on the LSM 710). AMCA fluorescence was imaged on the LSM 710 with excitation from a diode $405 \mathrm{~nm}$ laser, and selecting emission at $409-485 \mathrm{~nm}$. DyLight 649 fluorescence was imaged with excitation from a helium/neon $633 \mathrm{~nm}$ laser, with emission restricted by either a LP 650 filter (LSM 510) or by setting emission at $637-757 \mathrm{~nm}$ (LSM 710). A juxtacellularly labeled neuron was classified as not expressing the tested molecular marker only when positive immunoreactivity could be observed in other cells on the same focal plane as the tested neuron.

6-OHDA lesions of dopamine neurons. Unilateral 6-OHDA lesions were induced in $210-280 \mathrm{~g}$ rats, as detailed previously (Mallet et al., 2008a, b, 2012). The neurotoxin 6-OHDA (hydrochloride salt; Sigma) was dissolved in $0.9 \% \mathrm{w} / \mathrm{v} \mathrm{NaCl}$ solution containing $0.02 \% \mathrm{w} / \mathrm{v}$ ascorbate to a final concentration of $4 \mathrm{mg} / \mathrm{ml}$. Approximately $25 \mathrm{~min}$ before the injection of 6-OHDA, all animals received desipramine $(25 \mathrm{mg} / \mathrm{kg}$, i.p.; Sigma) to minimize the uptake of 6-OHDA by noradrenergic neurons (Schwarting and Huston, 1996b). Anesthesia was induced and maintained with isoflurane $\left(1.5 \%-3 \% \mathrm{v} / \mathrm{v}\right.$ in $\left.\mathrm{O}_{2}\right)$, and $3 \mu \mathrm{l}$ of 6-OHDA solution was injected near the medial forebrain bundle $(4.1 \mathrm{~mm}$ posterior and $1.2 \mathrm{~mm}$ lateral of Bregma, and $7.9 \mathrm{~mm}$ ventral to the dura) (Paxinos and Watson, 2007). Lesions were assessed 14 or $15 \mathrm{~d}$ after 6-OHDA injection by challenge with apomorphine (Schwarting and Huston, 1996a) $(0.05 \mathrm{mg} / \mathrm{kg}$, s.c.; Sigma) and were considered successful when animals made $\geq 80$ net contraversive rotations in $20 \mathrm{~min}$. Electrophysiological recordings were performed in the GPe ipsilateral to 6-OHDA lesions in anesthetized rats $21-35 \mathrm{~d}$ after surgery.

Analyses of in vivo electrophysiological data. Data from the recording session were visually inspected, and epochs of robust cortical SWA or cortical activation were selected according to the previously described characteristics of these brain states (Magill et al., 2006; Mallet et al., 2008a, b; Sharott et al., 2012). ECoGs were assumed to be realizations of stationary, zero-mean time series; their power spectra during SWA were calculated with a frequency resolution of $0.25 \mathrm{~Hz}$ using the MATLAB
(MathWorks) toolbox Neurospec 2.0 for multivariate Fourier analyses (www.neurospec.org). A portion of the spike train recorded during each defined brain state was isolated and used for statistical analyses (average epoch duration of $119 \pm 7 \mathrm{~s}$, mean \pm SEM). We assumed spike trains to be realizations of stationary, stochastic point processes. Putative singleunit activity was isolated with standard "spike sorting" procedures (Mallet et al., 2008a), including template matching, principal component analysis, and supervised clustering (Spike2). Isolation of a single unit was verified by the presence of a distinct refractory period in the interspike interval (ISI) histogram. Only neurons in which $<1 \%$ of all ISIs were $<2$ ms were analyzed in this study. For further analysis, single-unit activity was converted so that each spike was represented by a single digital event (Spike2). Mean firing rate (spikes per second; spk/s) was calculated from the total number of spikes per data epoch. The coefficient of variation of the ISIs $\left(\mathrm{CV}_{\text {ISI }}\right)$, a value used widely as an indicator of regularity in point processes (Johnson, 1996), and the SD of the ISIs $\left(\mathrm{SD}_{\text {ISI }}\right.$ ) were also calculated. To be included in the $\mathrm{CV}_{\text {ISI }}$ and $\mathrm{SD}_{\text {ISI }}$ analyses, a GPe neuron had to fire at least eight spikes during the in vivo recording.

To investigate how the spike firing of individual GPe neurons varied in time with respect to ongoing cortical slow oscillations during SWA, we used the Hilbert transform to analyze the instantaneous phase relationships between GPe spike times and cortical oscillations in a frequency band of 0.4-1.6 Hz (Sharott et al., 2012; Nakamura et al., 2014). Signal conditioning and analyses were performed using MATLAB (version 7.3; MathWorks). ECoG signals containing robust SWA were first filtered at $0.4-1.6 \mathrm{~Hz}$ using a second-order Butterworth filter. Subsequently, the instantaneous phase and power of the ECoG were calculated from the analytic signal obtained via the Hilbert transform (Lachaux et al., 1999; Le Van Quyen et al., 2001). In this formalism, peaks in the ECoG oscillations correspond to a phase of $0^{\circ}$ and troughs to a phase of $180^{\circ}$. Linear phase histograms, circular phase plots, and circular statistical measures were calculated using the instantaneous phase values for each spike. Descriptive and inferential circular statistics were then calculated using the CircStat toolbox (Berens, 2009) for MATLAB. For the calculation of vector lengths and statistical comparisons, we included only those GPe neurons that fired $\geq 40$ spikes during the entire recording during SWA. These neurons were then tested for significantly phase-locked firing (defined as having $p<0.05$ in Rayleigh's Uniformity Test). The null hypothesis for Rayleigh's Uniformity Test was that the spike data were distributed in a uniform manner. We and others have previously remarked that the nonsinusoidal nature of some field potential oscillations, such as the cortical slow oscillation, can confound standard circular statistics, especially Rayleigh's Uniformity Test (Siapas et al., 2005; Mallet et al., 2008a; Sharott et al., 2012; Nakamura et al., 2014). Thus, for analysis of GPe neuron firing relationships with cortical slow oscillations, Rayleigh's Uniformity Tests were only performed after any phase nonuniformities of the slow oscillations were corrected with the empirical cumulative distribution function of MATLAB (Siapas et al., 2005; Nakamura et al., 2014). For each of the neurons that were significantly phaselocked, the mean phase angle was calculated. Differences in the mean phase angles of groups of neurons were tested by the Watson-Williams $F$ test $(p<0.05$ for significance). The mean resultant vector length (referred to hereafter as simply "vector length") of the phase distribution, bound between 0 and 1 (the closer to 1 , the more concentrated the angles), was used to quantify the level of phase locking around the mean angle for individual neurons (computed using the angles of each spike) and for populations of neurons (computed using the mean angle for each neuron). Differences between the vector lengths of two neuron populations were tested for using the Mann-Whitney $U$ test, and differences between those of three populations with Kruskal-Wallis ANOVA on Ranks. Where data are displayed in circular plots (see Figs. 6 and 10), lines radiating from the center are the vectors of the preferred phases of firing (with the center and perimeter of the circle representing vector lengths of 0 and 1, respectively); thin lines indicate preferred firing of individual neurons, whereas thick black lines indicate population vectors. The small open circles on the perimeter represent the preferred phases of each neuron.

In vitro electrophysiological recording of individual GPe neurons. Visualized recordings of GPe neurons were performed in brain slices acutely 
prepared from 52 juvenile (P20-P35) dopamine-intact rats, as previously described (Baufreton et al., 2005, 2009; Miguelez et al., 2012). Briefly, animals were anesthetized with a mixture of ketamine and xylazine (75 $\mathrm{mg} / \mathrm{kg}$ and $10 \mathrm{mg} / \mathrm{kg}$, respectively, i.p.) and transcardially perfused with ice-cold modified "artificial CSF" (ACSF) that was equilibrated with $95 \%$ $\mathrm{O}_{2}$ and $5 \% \mathrm{CO}_{2}$ and contained the following (in $\mathrm{mM}$ ): 230 sucrose, 26 $\mathrm{NaHCO}_{3}, 2.5 \mathrm{KCl}, 1.25 \mathrm{Na}_{2} \mathrm{HPO}_{4}, 0.5 \mathrm{CaCl}_{2}, 10 \mathrm{MgSO}_{4}$, and 10 glucose. The brain was then quickly removed from the skull, blocked in the parasagittal plane, glued to the stage of a vibrating microtome (VT1200S; Leica Microsystems), and submerged in ice-cold modified ACSF. Slices containing the GPe (350 $\mu \mathrm{m}$ thick) were then cut and transferred to a holding chamber at room temperature in standard ACSF that was equilibrated with $95 \% \mathrm{O}_{2}$ and $5 \% \mathrm{CO}_{2}$, and contained the following (in $\mathrm{mm}$ ): $126 \mathrm{NaCl}, 26 \mathrm{NaHCO}_{3}, 2.5 \mathrm{KCl}, 1.25 \mathrm{Na}_{2} \mathrm{HPO}_{4}, 2 \mathrm{CaCl}_{2}, 2 \mathrm{MgSO}_{4}$, and 10 glucose. Single slices were then transferred to a recording chamber, perfused continuously with oxygenated ACSF at $35^{\circ} \mathrm{C}-37^{\circ} \mathrm{C}$, and visualized using infrared gradient contrast video microscopy (Eclipse workstation; Nikon) and a $60 \times$ water-immersion objective (Fluor $60 \times / 1.00 \mathrm{~W}$; Nikon). Recordings from individual GPe neurons were made in a cellattached or whole-cell configuration (voltage-clamp mode) or in a perforated-patch configuration (current clamp). In experiments designed to define the autonomous and driven firing of GPe neurons, all cell-attached and perforated-patch recordings were made in the presence of potent and selective blockers of glutamatergic synaptic transmission (50 $\mu \mathrm{M}$ APV and $20 \mu \mathrm{m}$ DNQX; Abcam) and GABAergic synaptic transmission (20 $\mu \mathrm{M}$ SR-95531/gabazine and $1 \mu \mathrm{M}$ CGP55845; Tocris Bioscience). Cell-attached recordings were made using standard-wall borosilicate glass electrodes filled with ACSF and were performed at a holding potential of $0 \mathrm{mV}$ after the stable establishment of the gigaseal. Somatic perforated-patch recordings were made using pipettes prepared from standard-wall borosilicate glass capillaries and were front-filled with the following (in mM): $110 \mathrm{KMeSO}_{4}, 25 \mathrm{KCl}, 3.6 \mathrm{NaCl}, 1 \mathrm{MgCl}_{2}$. $6 \mathrm{H}_{2} \mathrm{O}, 10$ HEPES, $0.1 \mathrm{Na}_{4}$ EGTA, $0.4 \mathrm{Na}_{3} \mathrm{GTP}$, and $2 \mathrm{Mg}_{1.5}$ ATP. The $\mathrm{pH}$ and osmolarity of the pipette solution were 7.3 and $290 \mathrm{mOsm}$, respectively. Patch pipettes were then back-filled with the same pipette solution containing gramicidin $(\sim 15 \mu \mathrm{g} / \mathrm{ml}$; Sigma). Gramicidin channels are only permeable to monovalent cations and small neutral molecules, allowing better preservation of the intrinsic physiological properties of GPe neurons, particularly their autonomous firing (Surmeier et al., 2005; Miguelez et al., 2012). For perforated-patch recordings, electrode capacitance was compensated online. Series resistance was regularly monitored, but not compensated, during recordings. On average, the series resistances during perforated-patch recordings of identified prototypic and arkypallidal neurons were similar $(69.4 \pm 4.9$ and $57.9 \pm 8.0 \mathrm{M} \Omega$, respectively). Voltage errors arising from series resistance were corrected off-line. Deliberate or accidental establishment of the whole-cell configuration was recognized as a sudden drop in series resistance and an offset $(\sim 5 \mathrm{mV})$ in membrane potential. The value of this offset was smaller than the experimentally measured, and the empirically calculated, junction potential between the electrode solution and the external media of 9 $\mathrm{mV}$ (Baufreton et al., 2005). The recorded membrane potential was therefore $\sim 4 \mathrm{mV}$ more depolarized than the true membrane potential and was corrected off-line. Somatic patch-clamp recordings were obtained using a Multiclamp 700B amplifier and Digitdata 1320A digitizer controlled by Clampex 9.0 software (Molecular Devices). Electrode signals were low-pass filtered at $4 \mathrm{kHz}$ and sampled at $20 \mathrm{kHz}$. Following electrophysiological recording in either a cell-attached or perforatedpatch configuration, the electrode was carefully withdrawn and the soma of the same neuron was repatched (Oren et al., 2009) using new glass pipettes filled with a solution containing $1.5 \% \mathrm{w} / \mathrm{v}$ biocytin (Sigma) and (in mM): $110 \mathrm{KMeSO}_{4}, 25 \mathrm{KCl}, 3.6 \mathrm{NaCl}, 1 \mathrm{MgCl}_{2} \cdot 6 \mathrm{H}_{2} \mathrm{O}, 10$ HEPES, 0.1 $\mathrm{Na}_{4}$ EGTA, $0.4 \mathrm{Na}_{3} \mathrm{GTP}$, and $2 \mathrm{Mg}_{1.5} \mathrm{ATP}$. The $\mathrm{pH}$ and osmolarity of this pipette solution were 7.2 and $290 \mathrm{mOsm}$, respectively. Subsequent establishment of a whole-cell recording configuration resulted in the rapid dialysis and anterograde filling of the GPe neuron with biocytin. This repatching procedure was used to increase the yield of biocytin-filled neurons after cell-attached or perforated-patch recordings of long duration; whole-cell dialysis with a pipette solution containing gramicidin can lead to widespread perforation of intracellular and plasma mem- branes, potentially killing the recorded neuron and, thence, precluding its biocytin labeling and post hoc verification. For measurements of "persistent" sodium channel currents $\left(\mathrm{I}_{\mathrm{NaP}}\right)$, neurons were recorded using a whole-cell voltage-clamp configuration in the presence of receptor blockers (as above), and held at $-95 \mathrm{mV}$ before and after the application of a slowly depolarizing ramp voltage command (from -80 to $-15 \mathrm{mV}$ ). The ramp command was set at $13.3 \mathrm{mV} / \mathrm{s}$, which reliably inactivates transient voltage-gated sodium channels, leaving the inward sodium current identified as $\mathrm{I}_{\mathrm{NaP}}$ (Park et al., 2013). Ramps were applied 3 times per neuron, and responses (peak amplitudes of $\mathrm{I}_{\mathrm{NaP}}$ ) were then averaged. Series resistance was regularly monitored, but not compensated, during recordings; if there was $>20 \%$ change in series resistance, neurons were excluded from further analyses. On average, the series resistances during whole-cell recordings of identified prototypic and arkypallidal neurons were similar ( $15.1 \pm 1.3$ and $15.6 \pm 0.7 \mathrm{M} \Omega$, respectively).

One to two hours after cell filling with biocytin, slices were immersed in $4 \% \mathrm{w} / \mathrm{v}$ PFA in PB for $1-7 \mathrm{~d}$ at $4^{\circ} \mathrm{C}$ and then cyroprotected in $20 \% \mathrm{w} / \mathrm{v}$ sucrose in PBS, embedded in Tissue-Tek (Fisher Scientific), frozen in isopentane (Sigma) at $-45^{\circ} \mathrm{C}$, and stored at $-80^{\circ} \mathrm{C}$ for $>2 \mathrm{~d}$ before resectioning.

Molecular characterization of GPe neurons recorded in vitro. Frozen slices were resectioned in the parasagittal plane at $50 \mu \mathrm{m}$ using a cryostat (CM3000; Leica Microsystems), collected in series, and washed in PBS. Endogenous peroxidases were inactivated by immersion of the freefloating sections in $3 \% \mathrm{v} / \mathrm{v} \mathrm{H}_{2} \mathrm{O}_{2}$ in PBS for $30 \mathrm{~min}$. Subsequently, sections were incubated in Triton PBS containing 1\% w/v BSA for $30 \mathrm{~min}$ and then incubated overnight at room temperature in Triton PBS containing AlexaFluor-568-conjugated streptavidin (1:500 dilution; Invitrogen). Biocytin-labeled neuronal somata were then isolated for molecular characterization by indirect immunofluorescence using the antibodies and protocols described above. Specifically, in vitro recorded and identified GPe neurons were tested for expression of FoxP2 and PV. After binding of primary and secondary antibodies, and final washing in PBS, sections were mounted in Vectashield and imaged on a confocal fluorescence microscope (TCS SP8; Leica Microsystems) equipped of a white light laser 2 with freely tunable excitation from 470 to $670 \mathrm{~nm}$.

Analyses of in vitro electrophysiological data. Data were analyzed using Origin 7.0 (Microcal Software). Mean firing rates, $\mathrm{CV}_{\text {ISI }}$, and $\mathrm{SD}_{\text {ISI }}$ were calculated as for recordings of GPe neurons in vivo. To quantify the autonomous firing of neurons recorded in the perforated-patch configuration, contiguous $60 \mathrm{~s}$ epochs of activity with zero current injection were analyzed. The input resistance of each neuron was calculated from the difference between the minimum membrane voltage reached during injection of a $-100 \mathrm{pA}, 500 \mathrm{~ms}$ current pulse, and the average subthreshold membrane potential during the $10 \mathrm{~s}$ preceding the current injection (Deister et al., 2013). As previously described (Baufreton et al., 2005), action potential threshold was determined using a custom algorithm that detected the first point of sustained positive "acceleration" of membrane voltage $[(\mathrm{d} V / \mathrm{d} t) / \mathrm{d} t]$ that was $>2$ SDs of baseline voltage before action potential threshold. Action potential half-width was calculated by measuring the action potential width at the midpoint between threshold and peak (Bugaysen et al., 2010). Frequency-current (F-I) response curves were obtained by injecting incremental current pulses from $-50 \mathrm{pA}$ to $200 \mathrm{pA}$ ( $2 \mathrm{~s}$ duration; $25 \mathrm{pA}$ steps). An index of firing rate adaptation was calculated as the ratio between the instantaneous firing frequencies of the first two spikes and last two spikes fired in response to a $2 \mathrm{~s}$ current pulse of 100 pA. "Rebound" burst firing was defined as spikes occurring at an instantaneous rate in excess of mean spontaneous firing +3 SDs in response to the injection of a $500 \mathrm{~ms}$ current pulse of $-150 \mathrm{pA}$. In voltage-clamp recordings, persistent sodium channel currents were transformed into conductance $(G)$ using Ohm's law in the form: $G=$ $\mathrm{I} /\left(\mathrm{V}-\mathrm{E}_{\mathrm{Na}}\right)$, where $\mathrm{V}$ is the test potential and $\mathrm{E}_{\mathrm{Na}}$ is the sodium equilibrium potential calculated using the Nernst equation. Conductance was normalized, plotted against $\mathrm{V}$, and fitted with a Boltzmann function of the form: $G / G_{\max }=1 /\left(1+\exp \left[\left(\mathrm{V}-\mathrm{V}_{1 / 2}\right) / k\right]\right)$, where $\mathrm{V}_{1 / 2}$ is the halfactivation voltage and $k$ is the slope factor.

Further statistical testing. The Shapiro-Wilk test was used to judge whether noncircular datasets were normally distributed ( $p \leq 0.05$ to reject). Because some datasets were not normally distributed, we used 
A

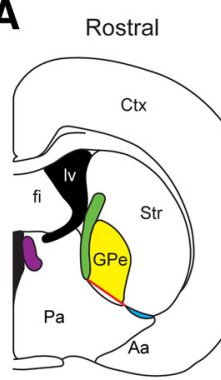

(Bregma $-0.6 \mathrm{~mm}$ )

C
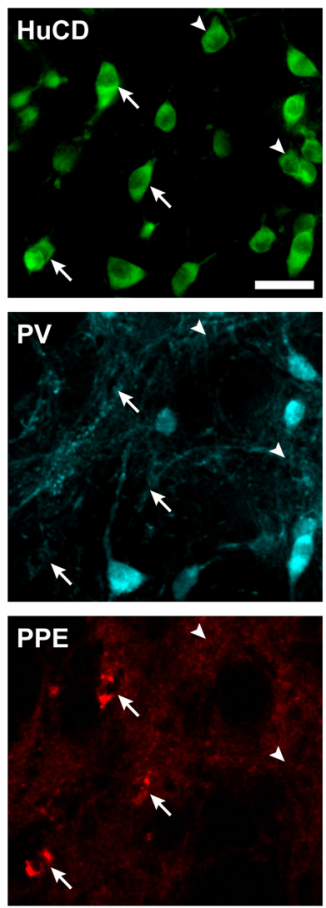

Central

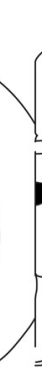

(Bregma $-1.0 \mathrm{~mm}$ )

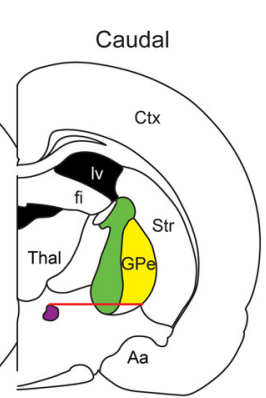

(Bregma -1.4 mm)

B

D

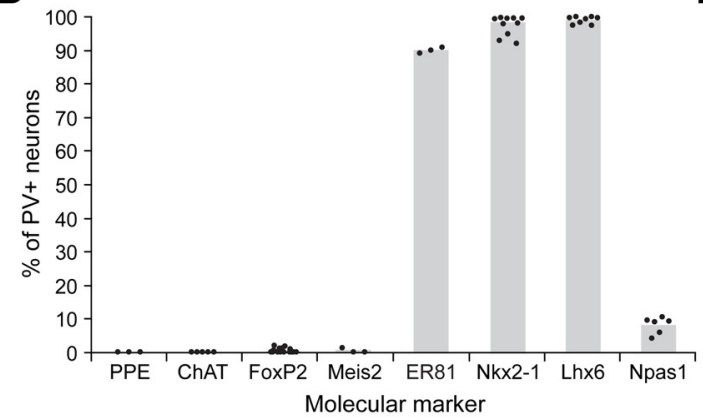

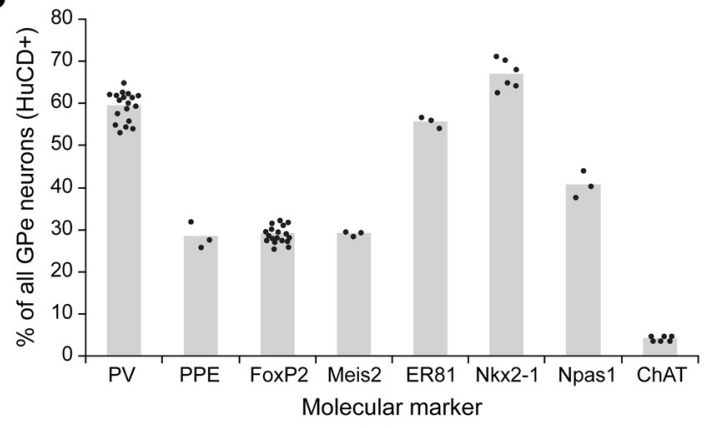

E
$\mathbf{F}$

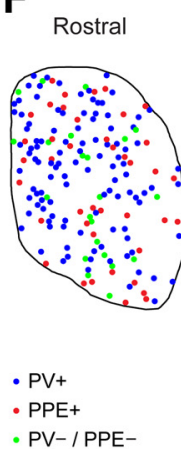

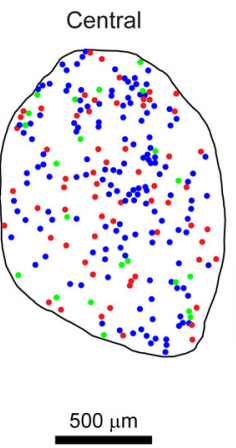

Caudal

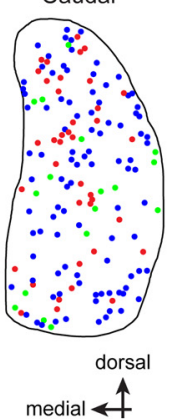

G

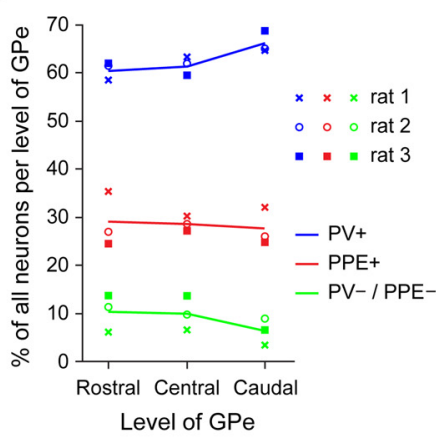

Figure 1. Most neurons of the dopamine-intact GPe express either PV or PPE. A, Coronal sections illustrating the rostral, central, and caudal levels of the adult rat GPe at which the molecular expression profiles of neurons were quantified. For GPe neuron sampling in rostral and central sections, the ventral borders of GPe (red lines) were defined according to the medial edge of the anterior commissure (blue) and the bottom edge of the internal capsule (green). In caudal sections, the ventral border of GPe was defined according to the fornix (purple). Only those neurons located dorsal to these borders were considered as GPe (yellow). Aa, Anterior amygdaloid area; Ctx, cortex; fi, fimbria of the hippocampus; Iv, lateral ventricle; Pa, preoptic area; Str, dorsal striatum; Thal, anterior thalamus. Adapted from Paxinos and Watson (2007). A standard stereotaxic reference (approximate distance posterior of Bregma) is given for each rostrocaudal level. $\boldsymbol{B}$, Proportions of all GPe neurons, defined with the pan-neuronal marker HuCD, expressing the given molecular markers (data pooled across rostral, central, and caudal GPe). In this and similar graphs, gray bars represent grand averages from all rats tested and each black dot represents the average from an individual rat. C, Immunofluorescence signals for HuCD, PV, and PPE in the GPe. Immunoreactivity for PPE was punctate and localized to the perikarya of neurons. PPE ${ }^{+}$GPe neurons (arrows) do not coexpress PV, and vice versa. A minority of GPe neurons do not express either PV or PPE (arrowheads). $\boldsymbol{D}$, Proportions of PV ${ }^{+}$GPe neurons coexpressing the given molecular markers. $\boldsymbol{E}$, Proportions of PPE ${ }^{+}$GPe neurons coexpressing the given molecular markers. $\boldsymbol{F}$, Maps showing distributions of PV ${ }^{+}$ neurons, $\mathrm{PPE}^{+}$neurons, and PV ${ }^{-} / \mathrm{PPE}{ }^{-}$neurons across the GPe (data from $10-\mu \mathrm{m}$-thick optical sections from one rat). The three cell types are distributed relatively evenly and are intermingled with each other. G, Prevalence of PV ${ }^{+}$neurons, PPE ${ }^{+}$neurons, and PV ${ }^{-} / \mathrm{PPE}^{-}$neurons at rostral, central, and caudal levels of GPe. Average proportions of these cell types in rats $1-3$ are indicated with colored lines. Scale bar: $C, 30 \mu \mathrm{m}$.

nonparametric statistical testing throughout (SigmaPlot 12; Systat Software). The Mann-Whitney $U$ test was used for comparisons of unpaired data, whereas the Wilcoxon Signed Rank Test was used to compare paired datasets. For multiple group comparisons, we performed a Kruskal-Wallis ANOVA on Ranks, with Dunn's Test for further post hoc definition of comparisons. Significance for all statistical tests was set at $p<0.05$ unless noted otherwise. Data are represented as group mean \pm SEM unless stated otherwise.

\section{Results}

Most GABAergic GPe neurons in dopamine-intact rats express either PV or PPE

In adult rats rendered parkinsonian by 6-OHDA lesions, most prototypic GPe neurons express the calcium-binding protein PV, whereas arkypallidal neurons instead express the neuropeptide precursor PPE (Mallet et al., 2012). We took these differences in the neurochemistry of prototypic and arkypallidal neurons in dopamine-depleted animals as a starting point for our investigation of GPe cell-type identity in dopamine-intact adult rats. We thus performed stereological analyses of immunofluorescence signals in rostral, central, and caudal GPe (Fig. 1A) to generate unbiased estimates of the proportions of neurons that express PV, PPE, both markers, or neither marker. In accordance with previous quantitative estimates of PV expression in adult rat GPe (Kita and Kitai, 1994; Kita and Kita, 2001; Hoover and Marshall, 2002), $\mathrm{PV}^{+}$neurons constituted $59 \%$ of all GPe neurons, the latter being identified here by immunoreactivity for the pan- 
A

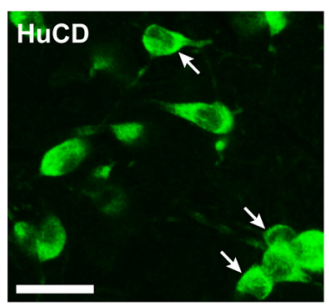

B

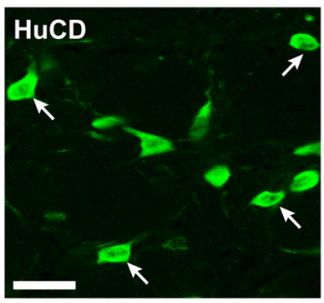

C

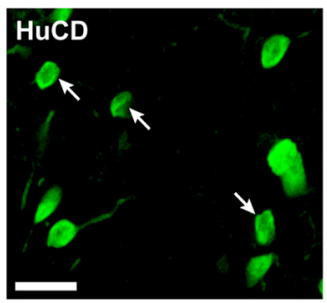

D

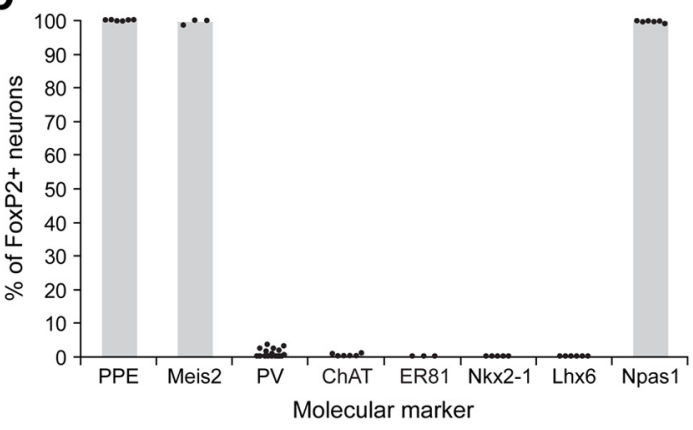

G
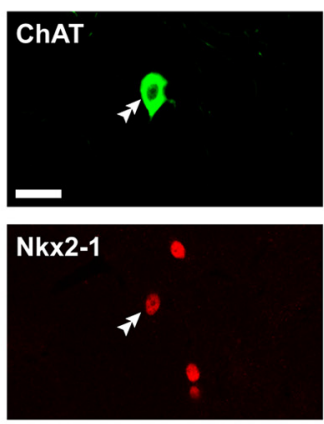
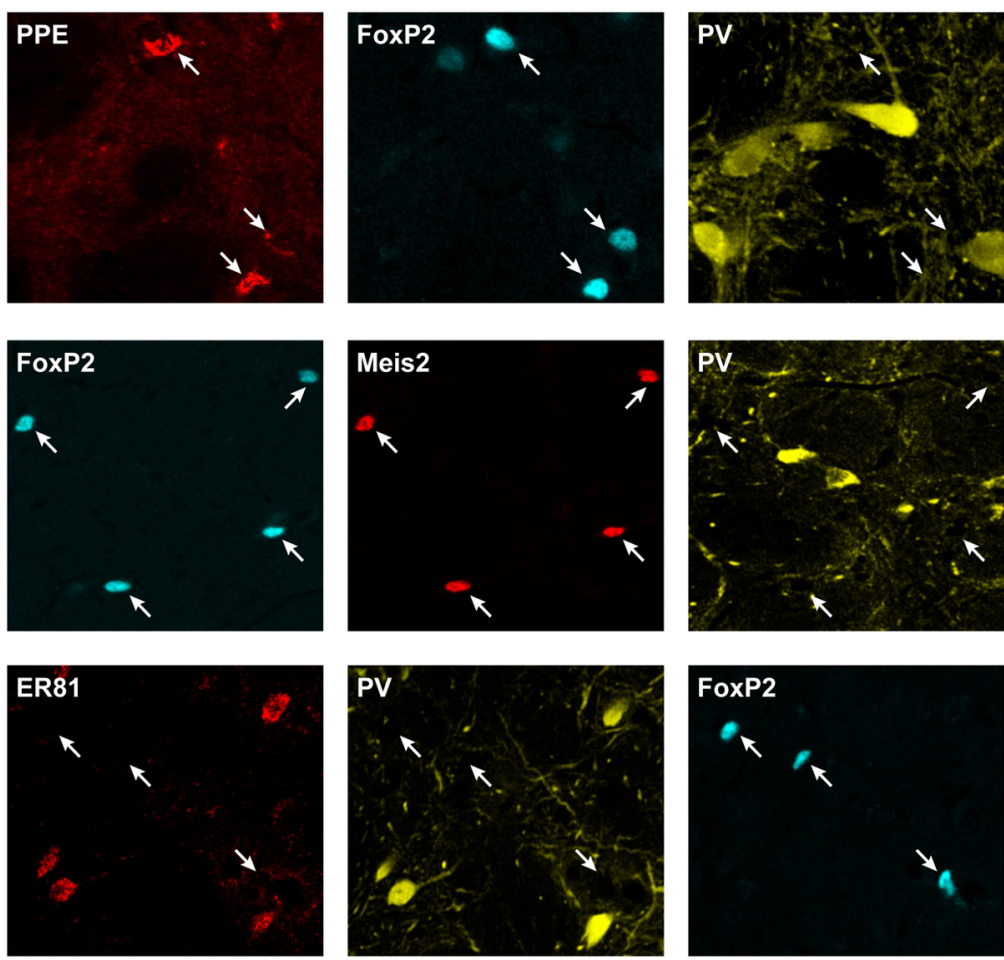

E

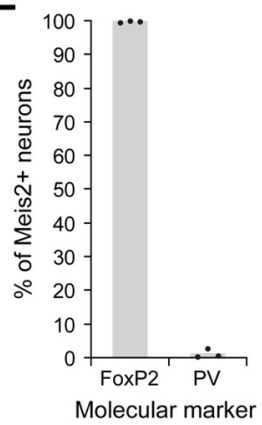

H

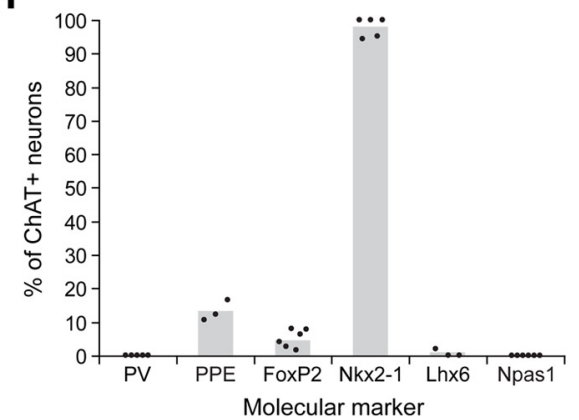

Figure 2. The neurochemical differences of the two major populations of GABAergic neurons in GPe tally with disparities in transcription factor expression. $A$, Immunofluorescence signals for HuCD, PPE, FoxP2, and PV in the GPe. Immunoreactivity for the transcription factor FoxP2 was localized to the nuclei of neurons. PPE ${ }^{+}$neurons coexpress FoxP2 but not PV (arrows). B, Signals for HuCD, FoxP2, Meis2, and PV in the GPe. Note the high level of coexpression of the transcription factors FoxP2 and Meis2 in neurons (arrows). C, Signals for HuCD, ER81, PV, and FoxP2 in the GPe. The transcription factor ER81 was localized to the cytoplasm and nuclei of GPe neurons. Note the high level of coexpression of ER81 and PV, and that FoxP2 ${ }^{+}$neurons (arrows) do not coexpress ER81.D, Proportions of FoxP2 ${ }^{+} \mathrm{GPe}$ neurons coexpressing a given molecular marker. E, Proportions of Meis $2^{+}$GPe neurons coexpressing FoxP2 or PV.F, Proportions of ER81 ${ }^{+}$GPe neurons coexpressing PV or FoxP2.G, Signals for ChAT, the transcription factor Nkx2-1, FoxP2, and PV in the GPe. MostChAT ${ }^{+}$neurons (double arrowhead) coexpress Nkx2-1, but notPV or FoxP2. H, Proportions of ChAT ${ }^{+}$GPeneurons coexpressing a given molecular marker. Scale bars: $A-C$, G, $30 \mu \mathrm{m}$.

neuronal marker $\mathrm{HuCD}$ (Fig. $1 B, C)$. On average, $\mathrm{PPE}^{+}$neurons were less numerous than $\mathrm{PV}^{+}$neurons but still constituted over one-fourth (28\%) of all GPe neurons (Fig. 1B). Importantly, coexpression of PV and PPE by individual GPe neurons was negligible $\left(<0.2 \%\right.$ of $\mathrm{PV}^{+}$neurons expressed PPE and vice versa; Fig.
$1 C-E)$. However, a substantial proportion of all GPe neurons (10\%) expressed neither PV nor PPE ( $\mathrm{PV}^{-} / \mathrm{PPE}^{-}$neurons; Fig. $1 B, C)$. Cholinergic GPe neurons, identified by immunoreactivity for ChAT, comprised $4 \%$ of all GPe neurons (Fig. 1B). Coexpression of ChAT and PV was not observed (Figs. $1 D$ and $2 H$ ). A 
small proportion $(13 \%)$ of $\mathrm{ChAT}^{+}$neurons coexpressed PPE (Fig. $2 \mathrm{H}$ ), but, because cholinergic GPe neurons are rare, this meant that $<1 \%$ of $\mathrm{PPE}^{+}$neurons coexpressed ChAT (Fig. 1E). Moreover, the scarcity of cholinergic neurons indicates that they could only account for a minority of $\mathrm{PV}^{-} / \mathrm{PPE}^{-}$neurons in the $\mathrm{GPe}$ regions that were analyzed. $\mathrm{PV}$-expressing neurons, $\mathrm{PPE}^{+}$ neurons, and $\mathrm{PV}^{-} / \mathrm{PPE}^{-}$neurons were distributed throughout GPe and were intermingled with each other (Fig. 1C,F). On average, the proportions of all GPe neurons that were $\mathrm{PV}^{+}, \mathrm{PPE}^{+}$, or $\mathrm{PV}^{-} / \mathrm{PPE}^{-}$were relatively constant across the rostral, central, and caudal levels of GPe (Fig. $1 G$ ). Together, these data show that most GPe neurons express either PV or PPE. However, assuming that all noncholinergic GPe neurons in rodents and primates use GABA as their primary neurotransmitter (Smith et al., 1987; Delfs et al., 1995), our data also show that PV and PPE do not collectively capture all GABAergic GPe neurons.

\section{The two major populations of GABAergic GPe neurons in dopamine-intact rats are delineated by their selective expression of transcription factors}

A specific molecular marker for prototypic GPe neurons has not yet been determined (Mallet et al., 2012). Moreover, PPE is the only marker known to distinguish arkypallidal neurons from prototypic neurons (Mallet et al., 2012). There is thus a pressing need to identify other molecular markers that more comprehensively define these two cell types in the dopamine-intact GPe. With this in mind, we interrogated the published literature and other online resources (Allen Brain Atlas; http://www.brain-map.org/) and judged six transcription factors (FoxP2, Meis2, ER81, Nkx2-1, Lhx6, and Npas1) to be especially promising candidate markers. We then used the same stereological sampling approach to quantify their (co)expression in $\mathrm{PPE}^{+}, \mathrm{PV}^{+}$, and $\mathrm{PV}^{-} / \mathrm{PPE}^{-}$neuronal populations. Neurons expressing PPE, FoxP2, or Meis2 were similarly abundant in GPe (Fig. 1B), and critically, there was an almost perfect overlap in the expression of these three markers. Indeed, $>99 \%$ of $\mathrm{PPE}^{+}$neurons expressed FoxP2 and vice versa (Figs. $1 E$ and $2 A, D)$, and $>99 \%$ of FoxP $2^{+}$neurons expressed Meis 2 and vice versa (Fig. $2 B, D, E$ ). In stark contrast, coexpression of FoxP2 (or Meis2) and PV was extremely rare, such that $<1 \%$ of FoxP2 ${ }^{+} /$ Meis $2^{+}$neurons expressed PV and vice versa (Figs. $1 D$ and $2 A, B, D, E)$. Coexpression of ChAT by FoxP2 ${ }^{+}$neurons was similarly rare (Fig. $2 D$ ). These data suggest that arkypallidal neurons are not only defined by their expression of PPE but also by expression of FoxP2 and/or Meis2. However, it follows that these two transcription factors do not mark the $\mathrm{PV}^{-} / \mathrm{PPE}^{-}$GABAergic neuron population in GPe.

Studies of the embryonic and early postnatal development of mouse ventral telencephalon (subpallium) show that the molecular diversity of GPe neurons extends to the selective expression of other transcription factors that are critical for the genesis, migration, specification, and maturation of neurons (Flandin et al., 2010; Nóbrega-Pereira et al., 2010). For example, expression of the ETS transcription factor ER81 informs on the progenitor domains of the embryonic subpallium that give rise to GPe neurons (Flames et al., 2007; Flandin et al., 2010; Nóbrega-Pereira et al., 2010). We determined that, on average, ER $81^{+}$neurons constituted $56 \%$ of all GPe neurons in dopamine-intact adult rats (Fig. 1B). Coexpression of ER81 and PV in individual GPe neurons was common; almost all ER $81^{+}$neurons (97\%) coexpressed $\mathrm{PV}$, and $90 \%$ of $\mathrm{PV}^{+}$neurons coexpressed ER81 (Figs. $1 D$ and $2 C, F)$. However, ER81 ${ }^{+}$GPe neurons did not express FoxP2 (Fig. 2C,D,F), a proxy marker for $\mathrm{PPE}^{+} \mathrm{GPe}$ neurons (see above). Thus, although the selective expression of ER81 further resolves the molecular phenotype of adult rat GPe neurons, it is largely comparable with that of PV.

Because many neurons in normal mouse GPe express the homeobox transcription factor Nkx2-1 (Magno et al., 2009; Nóbrega-Pereira et al., 2010) and because mutant mice with disrupted $N k x 2-1$ gene function lack a readily discernible GPe (Sussel et al., 1999; Flandin et al., 2010), we next quantified the expression of Nkx2-1 in the GPe of dopamine-intact adult rats. On average, $\mathrm{Nkx} 2-1^{+}$neurons constituted approximately twothirds $(67 \%)$ of all GPe neurons (Fig. 1B). Although a small fraction $(5 \%)$ of these Nkx2-1 ${ }^{+}$neurons coexpressed ChAT (Figs. $2 G$ and $3 E$ ), the majority of $\mathrm{Nkx} 2-1^{+}$neurons $(86 \%)$ coexpressed PV instead (Fig. $3 A, E)$. However, almost all (98\%) $\mathrm{PV}^{+}$neurons coexpressed Nkx2-1 (Figs. $1 D$ and $3 A$ ). Thus, although there is a large overlap in the expression of $\mathrm{Nkx} 2-1$ and $\mathrm{PV}$, these data further highlight that GABAergic $\mathrm{Nkx2}-1^{+}$GPe neurons are more abundant than GABAergic $\mathrm{PV}^{+} \mathrm{GPe}$ neurons. Importantly, Nkx2-1 ${ }^{+}$GPe neurons did not express FoxP2 (Figs. 2D and $3 A, E)$.

We also analyzed the expression of the transcription factor Lhx6, which acts downstream of Nkx2-1 to direct the migration and specification of several telencephalic cell types, including some GPe neurons (Sussel et al., 1999; Liodis et al., 2007; Du et al., 2008; Flandin et al., 2010). In line with the requirement of $N k \times 2-1$ for the expression of $\operatorname{Lhx} 6$ (Sussel et al., 1999; Butt et al., 2008; Flandin et al., 2010), coexpression of Nkx2-1 and Lhx6 in individual GPe neurons was very common. Indeed, $96 \%$ of $\mathrm{Nkx} 2-1^{+}$ neurons also expressed Lhx6, and 97\% of Lhx6 ${ }^{+}$neurons also expressed Nkx2-1 (Fig. 3 B,E,F). Virtually all (99\%) $\mathrm{PV}^{+}$neurons coexpressed Lhx6, whereas only $86 \%$ of Lhx6 ${ }^{+}$neurons coexpressed PV (Figs. $1 D$ and $3 B, F$ ). Coexpression of Lhx6 and ChAT in GPe neurons was exceedingly rare (Figs. $2 \mathrm{H}$ and $3 F$ ). As could be expected from the near-equivalent expression profiles of Lhx6 and Nkx2-1 in GABAergic GPe neurons, coexpression of Lhx6 and FoxP2 was not observed (Figs. $2 D$ and $3 B, F$ ). Because Nkx2-1 ${ }^{+} / \mathrm{Lhx}^{+}$neurons and $\mathrm{PPE}^{+} / \mathrm{FoxP}^{+}$neurons accounted for $\sim 68 \%$ and $30 \%$ of GABAergic GPe neurons, respectively, the expression of Nkx2-1 and/or Lhx6 ostensibly captures the remaining $\mathrm{PV}^{-} / \mathrm{PPE}^{-}$GABAergic neuron population. These data collectively suggest that, within a framework of dichotomous cell types in adult GPe, Nkx2-1 and Lhx6 can be used interchangeably as both comprehensive and highly selective markers for GABAergic prototypic neurons.

The basic helix-loop-helix transcription factor Npas1 has been recently shown to mark a novel population of GABAergic cells in mouse GPe, although reports disagree about the prevalence and extended molecular profile of these Npas ${ }^{+}$neurons (Flandin et al., 2010; Nóbrega-Pereira et al., 2010). We found that, on average, $\mathrm{Npas}^{+}$neurons constituted $41 \%$ of all GPe neurons in adult rats (Fig. $1 B$ ). The majority $(71 \%)$ of $\mathrm{Npas}^{+}$ GPe neurons coexpressed FoxP2 (Fig. 3C,D, G), whereas virtually all $(>99 \%)$ FoxP2 $^{+}$neurons coexpressed Npas1 (Fig. 2D). Most Npas $1^{+} / \mathrm{FoxP}^{-}$neurons coexpressed Lhx6 and Nkx2-1, and approximately one-half of these neurons additionally expressed PV (Fig. 3D, G). Npas1-expressing neurons comprised 15\% of Lhx6 ${ }^{+}$neurons (Fig. 3F), 13\% of Nkx2-1 ${ }^{+}$neurons (Fig. 3E), and $8 \%$ of $\mathrm{PV}^{+}$neurons (Fig. 1D). Coexpression of Npas1 and ChAT was not observed (Figs. $2 H$ and $3 G$ ). These data show that Npas 1 is expressed by at least three molecularly distinct GABAergic cell types in GPe and that Npas 1 expression cannot be used to neatly discriminate FoxP2 ${ }^{+}$arkypallidal neurons from $\mathrm{Nkx} 2$ $1^{+} /$Lhx $6^{+}$prototypic neurons per se. 


\section{A}

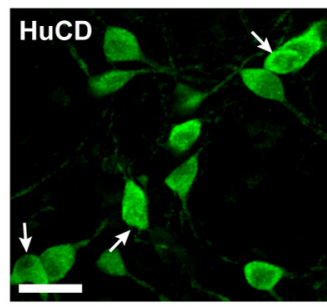

B

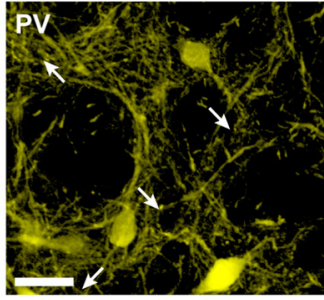

C

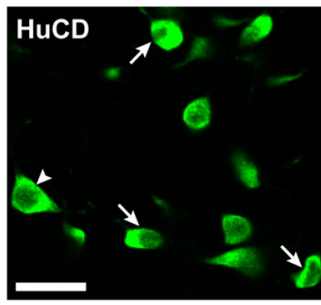

D

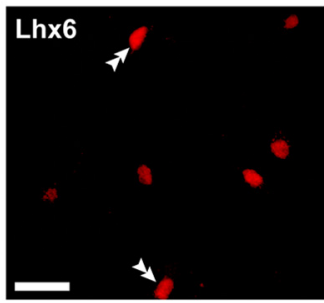

E

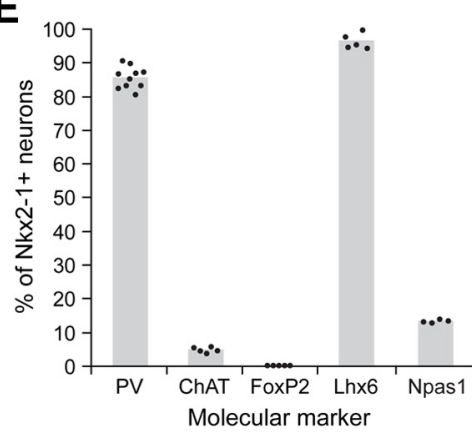

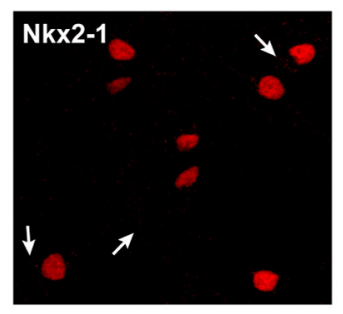
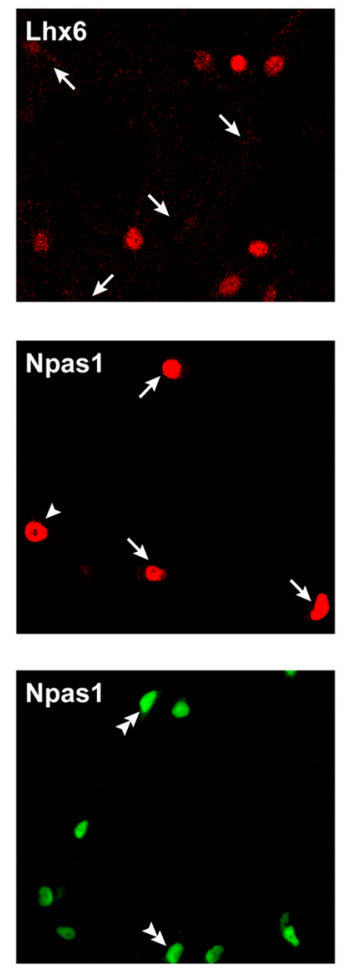

F

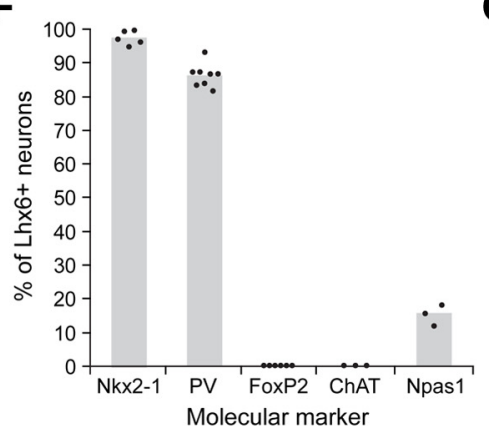

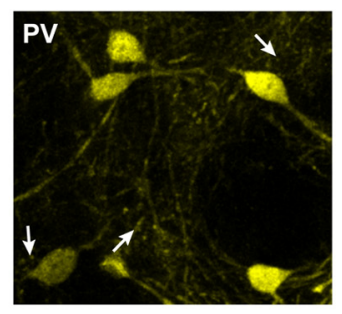
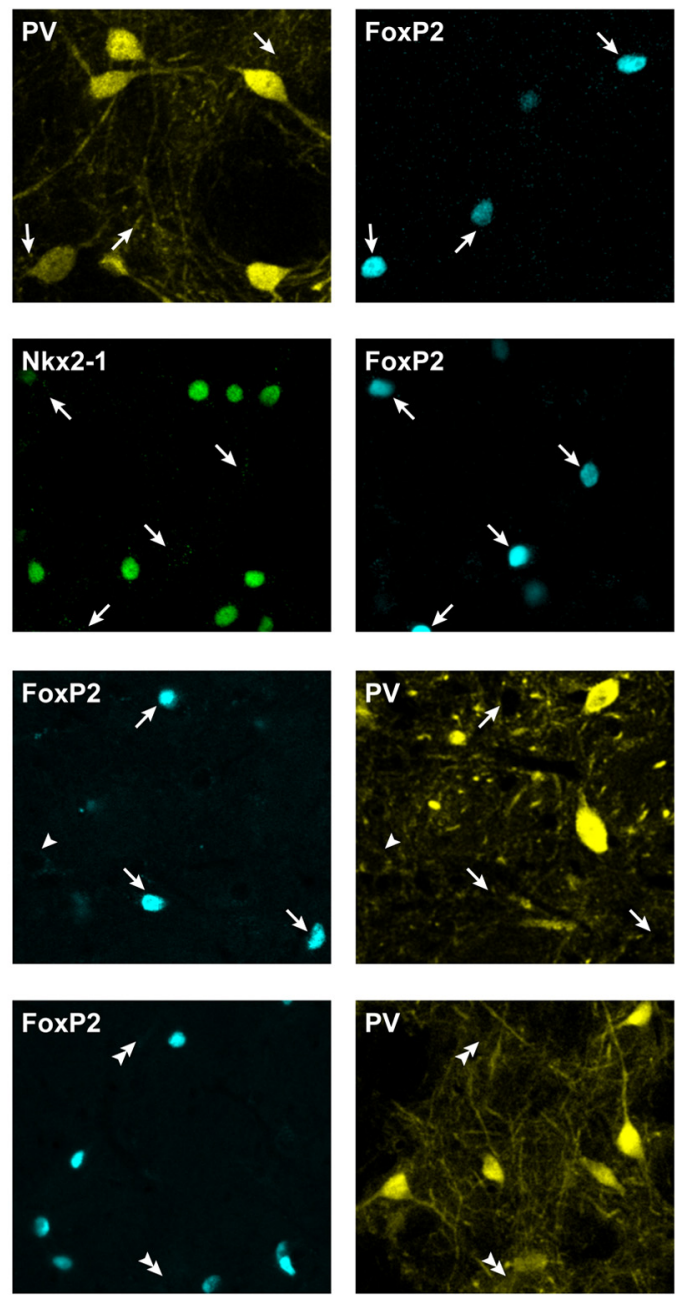

G

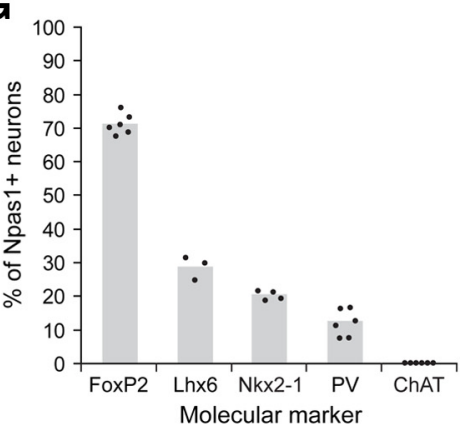

Figure 3. The two major populations of GABAergic neurons in GPe are distinguished by their mutually exclusive expression of the transcription factors Nkx2-1/Lhx6 or FoxP2. A, Immunofluorescence signals for HuCD, Nkx2-1, PV, and FoxP2 in the GPe. Most Nkx2-1 ${ }^{+}$neurons are also PV ${ }^{+}$. FoxP2 ${ }^{+}$neurons (arrows) do not coexpress Nkx2-1. B, Signals for PV, Lhx6, Nkx2-1, and FoxP2 in the GPe. Note the high level of coexpression of the transcription factors Lhx6 and Nkx2-1. FoxP2 ${ }^{+}$neurons (arrows) do not coexpress Lhx6. C, Signals for HuCD, Npas1, FoxP2, and PV in the GPe. Most Npas $1^{+}$neurons coexpress FoxP2 (arrows) but not PV. Some Npas $1^{+}$neurons do not express either marker (arrowhead). D, Signals for Lhx6, Npas1, FoxP2, and PV in the GPe. Most $\mathrm{Npas}^{+}{ }^{+}$/FoxP2 ${ }^{-}$neurons also express Lhx6 (double arrowheads). $\boldsymbol{E}$, Proportions of Nkx2-1 ${ }^{+}$GPe neurons coexpressing a given molecular marker. $\boldsymbol{F}$, Proportions of Lhx6 ${ }^{+} \mathrm{GPe}$ neurons coexpressing a given molecular marker. G, Proportions of Npas $1^{+}$GPe neurons coexpressing a given molecular marker. Scale bars: A-D, $30 \mu \mathrm{m}$.

Pallidosubthalamic neurons in dopamine-intact rats express Nkx2-1 and PV but not FoxP2

In dopamine-depleted rats, prototypic GPe neurons can be further distinguished from arkypallidal neurons on the basis of their structure and, particularly, by their long-range axonal projections. As a working definition, arising from a modest sample of individually recorded, labeled, and reconstructed neurons (Mallet et al., 2012), all prototypic neurons innervate at least one of the "downstream" BG nuclei, such as the STN, whereas arkypallidal neurons innervate only the striatum. When considering these structural differences in light of the molecular expression profiles we detail above for large GPe cell populations, one might expect that, in dopamine-intact animals, Nkx2-1 ${ }^{+} /$Lhx6 ${ }^{+}$neurons constitute the majority of GPe neurons innervating the STN, whereas FoxP $2{ }^{+}$GPe neurons innervate the striatum but not the STN. To test these two predictions, we retrogradely transduced GPe neurons innervating either STN or striatum with a lentiviral vector expressing EGFP (Kato et al., 2007) and then quantified the pro- 
A

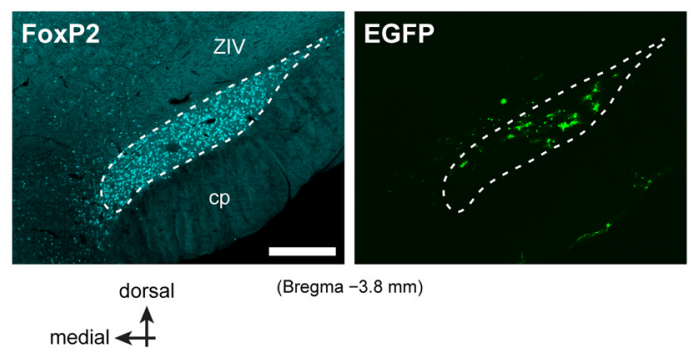

c
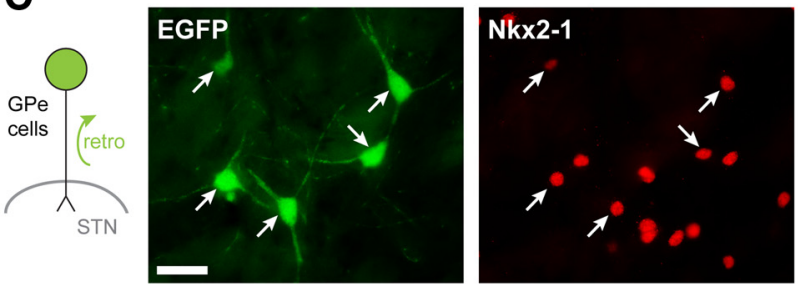

D
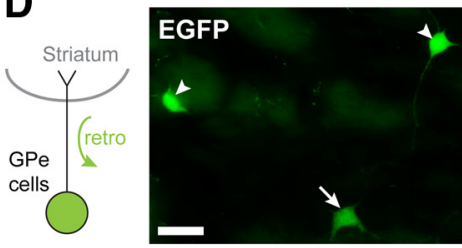

E
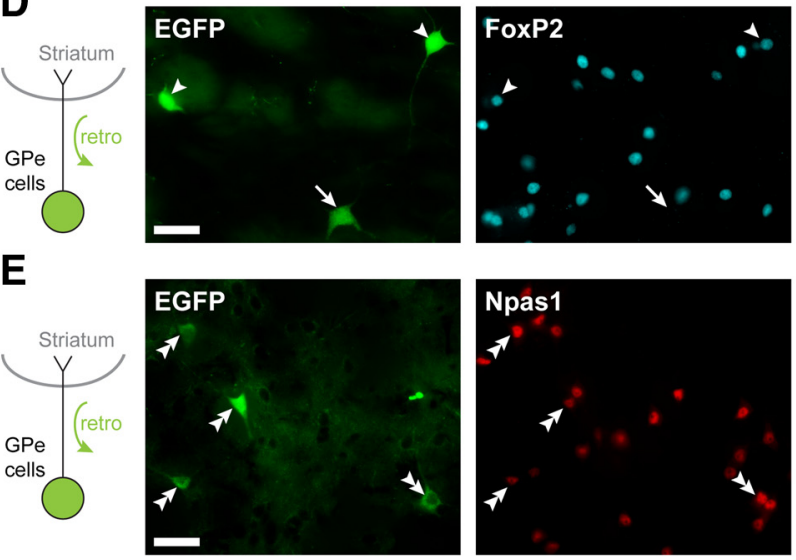

G

F

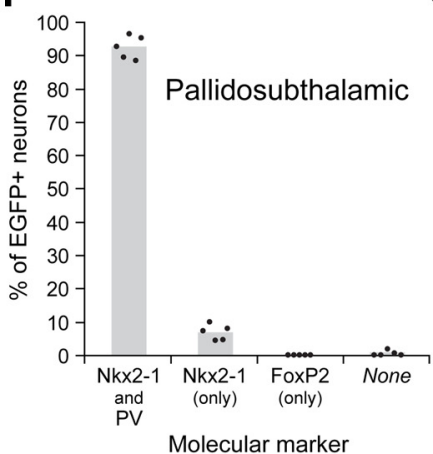

B
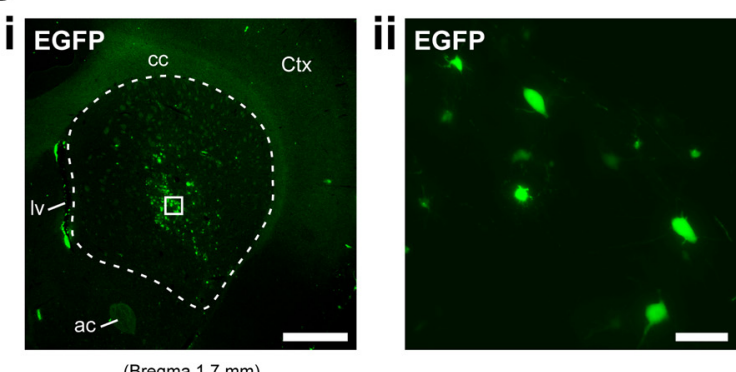

(Bregma $1.7 \mathrm{~mm}$ )
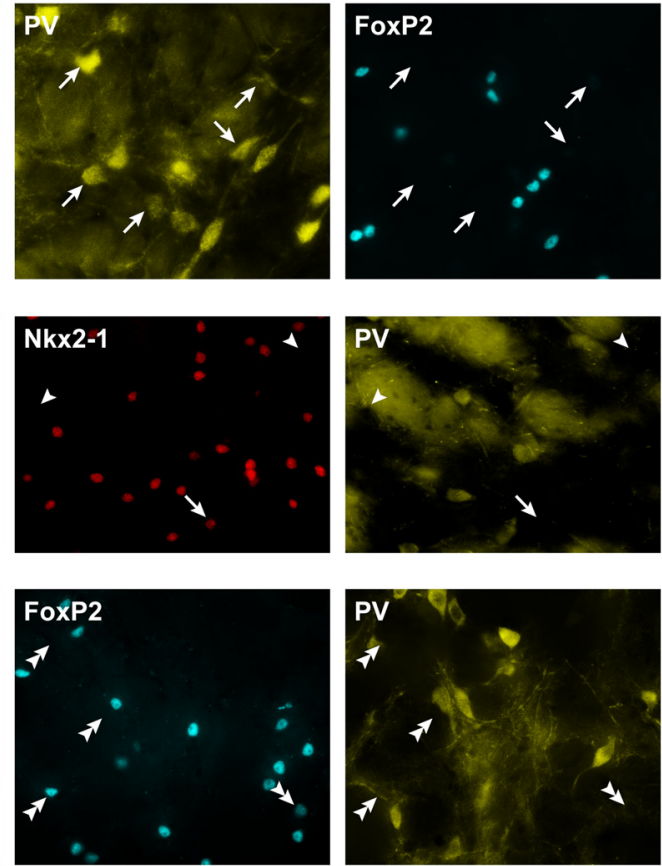

H
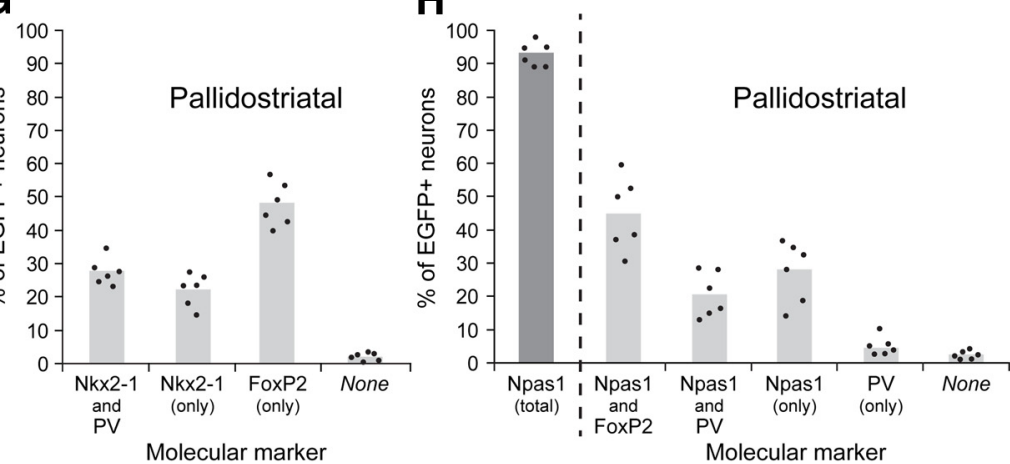

Figure 4. Pallidosubthalamic and pallidostriatal neurons are differentiated by their proportional expression of distinct sets of molecular markers. $A, B$, Verification of sites of injection of a lentiviral vector expressing EGFP into the STN $(\boldsymbol{A})$ or dorsal striatum (B). $\boldsymbol{A}$, Left, The STN was delineated according to the boundaries (dashed lines) of a group of densely packed neurons immunoreactive for FoxP2. Right, EGFP-expressing cells in the same coronal section. Bi, Low-magnification image ofEGFP ${ }^{+}$cells in a central region of dorsal striatum (within dashed lines). Bii, Higher-magnification image of EGFP ${ }^{+}$neurons and glia within the area boxed with white lines in Bi. ac, Anterior commissure; $c c$, corpus callosum; $c$, cerebral peduncle; $C \mathrm{Ctx}$, cortex; Iv, lateral ventricle; ZIV, ventral division of the zona incerta. Approximate distances posterior/anterior to Bregma are given. C, Left, GPe neurons innervating the STN were identified by their expression of EGFP after retrograde viral transduction (retro). Most of these EGFP ${ }^{+}$pallidosubthalamic neurons (white arrows) coexpress Nkx2-1 and PV. However, identified pallidosubthalamic neurons do not express FoxP2. D, Many GPe neurons innervating the dorsal striatum, as identified by EGFP expression after separate retrograde viral transduction, coexpress FoxP2 (arrowheads). Another major population of identified pallidostriatal neurons expresses Nkx2-1 (white arrows). $\boldsymbol{E}$, Most identified pallidostriatal neurons express Npas1 (double arrowheads).F, Proportions of EGFP ${ }^{+}$pallidosubthalamicneurons that coexpress the given molecular marker or combination of markers. Virtually all pallidosubthalamic neurons express Nkx2-1.G, $\boldsymbol{H}$, Proportions of EGFP ${ }^{+}$pallidostriatal neurons coexpressing the given molecular marker or combination of markers. Approximately one-half of pallidostriatal neurons express FoxP2 (G), but the vast majority express Npas1 $(\boldsymbol{H}) \cdot \boldsymbol{H},{ }^{\prime}$ "Npas1 (total)" refers to the sum of Npas $1^{+} / \mathrm{FoxP2}^{+}, \mathrm{Npas}^{+} / \mathrm{PV}^{+}$, and Npas $1^{+}$(only) populations of transduced neurons. Scale bars: $\boldsymbol{A}, 400 \mu \mathrm{m} ; \boldsymbol{B i}, 1 \mathrm{~mm} ; \boldsymbol{B i i ,} \mathbf{C}-\boldsymbol{E}, 30 \mu \mathrm{m}$.

portions of $\mathrm{EGFP}^{+}$neurons expressing Nkx2-1, PV, and/or FoxP2. Injections of the lentiviral vector into the STN (Fig. $4 A$ ) resulted in robust somatodendritic expression of EGFP by GPe neurons (Fig. 4C). The vast majority (99\%) of sampled EGFP ${ }^{+}$
GPe neurons innervating STN ( $n=377$ neurons from 5 rats) coexpressed Nkx2-1 (Fig. 4C,F). Most (93\%) of these EGFP ${ }^{+} /$ Nkx2 $-1^{+}$neurons additionally expressed PV (Fig. 4C,F). However, $\mathrm{EGFP}^{+}$pallidosubthalamic neurons did not express FoxP2 
A

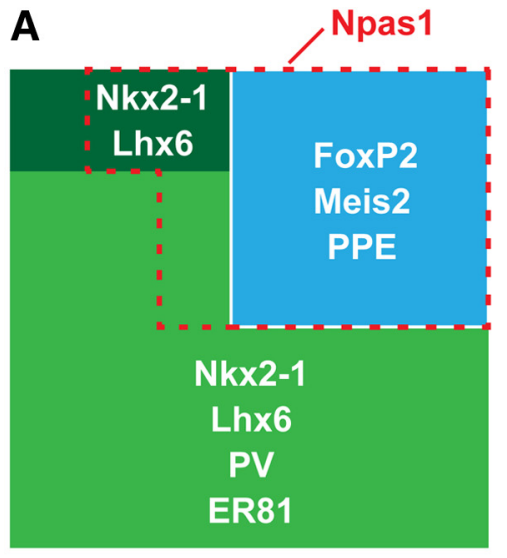

\section{Arkypallidal neurons}

\section{Prototypic neurons}

B

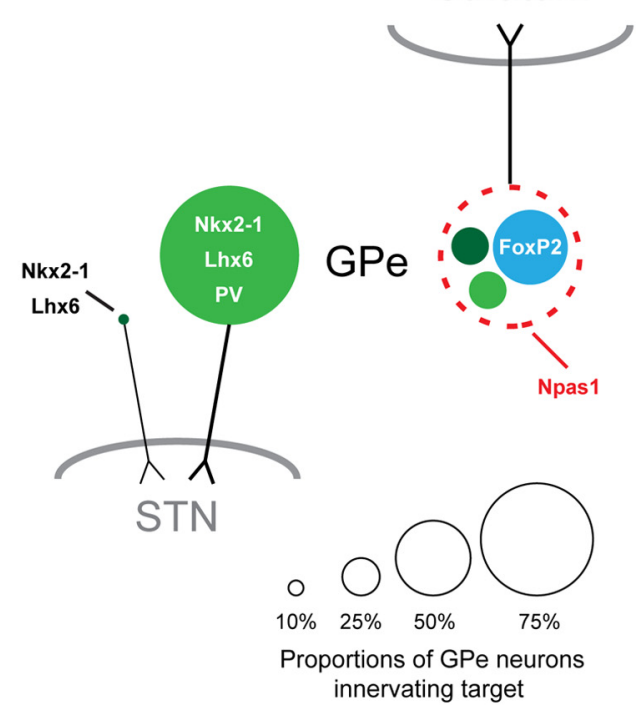

Figure 5. Molecular signatures and projection targets of prototypic neurons and arkypallidal neurons. $\boldsymbol{A}$, Schematic summary of the two major GABAergic cell types in the GPe of the rat. Colored areas are scaled to show the relative population sizes of arkypallidal neurons (blue) and prototypic neurons (light and dark greens) with respect to all GABAergic GPe neurons. The distinct sets of molecular markers that identify each cell type are indicated. However, both arkypallidal neurons and some prototypic neurons express Npas 1 (within red dashed line). B, Schematic summary of the major GPe cell types innervating the STN (left) or dorsal striatum (right). Almost all pallidosubthalamic neurons are of the prototypic cell type. The vast majority of pallidostriatal neurons express Npas1. Arkypallidal neurons do not innervate STN but are the major GPe cell type innervating striatum.

neurons were Nkx2-1 ${ }^{+} /$Lhx6 ${ }^{+}$(Fig. $3 G$, and compare Fig. $4 G, H)$. These data show that Npas1 is a highly selective molecular marker for GABAergic pallidostriatal neurons, regardless of arkypallidal or prototypic cell-type identities.

\section{Molecular signatures and projection targets of prototypic neurons and arkypallidal neurons in the dopamine- intact rat GPe}

Data from our cell counting and retrograde tract-tracing experiments converge to show that prototypic and arkypallidal neurons are optimally and comprehensively delineated by their expression of different sets of transcription factors (Fig. 5). Prototypic neurons are defined by their expression of $\mathrm{Nkx2}-1$ and Lhx6; $\sim 85 \%$ of prototypic neurons additionally coexpress PV and ER81 (Fig. 5A). Arkypallidal neurons are defined by their coexpression of FoxP2 and Meis2; they also express PPE (Fig. 5A). Together, prototypic and arkypallidal neurons account for $>98 \%$ of all GABAergic GPe neurons. Virtually all GPe neurons innervating STN are of the prototypic cell type (Fig.

(Fig. 4C,F). These data confirm that $\mathrm{Nkx} 2-1^{+}\left(\mathrm{PV}^{+}\right)$neurons are indeed the major GPe cell population innervating STN, thus further ascribing the prototypic cell-type identity to them, and also show that $\mathrm{FoxP}_{2}{ }^{+}$GPe neurons do not innervate STN. Injections of the lentiviral vector into the dorsal striatum of 6 other rats (Fig. $4 B$ ) also resulted in $\mathrm{EGFP}^{+}$GPe neurons (Fig. $4 D$ ), but their predominant molecular phenotypes differed from those innervating STN. Indeed, on average, approximately one-half (48\%) of sampled $\mathrm{EGFP}^{+} \mathrm{GPe}$ neurons innervating striatum $(n=390$ neurons from 6 rats) coexpressed FoxP2, but not Nkx2-1 or PV (Fig. $4 D, G)$. Almost all $(96 \%)$ of the remaining $\mathrm{EGFP}^{+} / \mathrm{FoxP}^{-}$ pallidostriatal neurons coexpressed Nkx2-1; and, of these, approximately one-half also expressed PV (Fig. 4D, G). These data collectively show that FoxP2 ${ }^{+}$GPe neurons innervate striatum but not STN, thus further ascribing the arkypallidal identity to them. Furthermore, FoxP2 ${ }^{+}$arkypallidal neurons are the major GPe cell type innervating the central regions of dorsal striatum, with smaller cohorts of Nkx2-1 ${ }^{+} / \mathrm{PV}^{-}$neurons and $\mathrm{Nkx} 2-1^{+} /$ $\mathrm{PV}^{+}$neurons making up the remainder of the pallidostriatal cell population.

In accordance with previous work (Bevan et al., 1998; Mallet et al., 2012), our data show that both arkypallidal neurons and a minor population of prototypic neurons contribute to the pallidostriatal projection as a whole. Thus, pallidostriatal identity does not tally with a molecular dichotomy defined on the basis of a mutually exclusive expression of FoxP2 or Nkx2-1/Lhx6. Given that Npas1 was not only expressed by virtually all FoxP2 ${ }^{+}$neurons but also by a minority of Nkx2-1 $1^{+} / \mathrm{Lhx} 6^{+}$neurons (Figs. $2 D$ and $3 C-F$ ), we next quantified the relationship between Npas1 expression and pallidostriatal identity. Of the $\mathrm{EGFP}^{+} \mathrm{GPe}$ neurons innervating striatum that were tested for Npas1, FoxP2, and PV ( $n=177$ neurons), the vast majority (93\%) coexpressed Npas1 (Fig. $4 E, H$ ). Of these $\mathrm{EGFP}^{+} / \mathrm{Npas}^{+}{ }^{+}$neurons, $48 \%$ were FoxP2 ${ }^{+}$and $22 \%$ were instead $\mathrm{PV}^{+}$(Fig. $4 E, H$ ). It is likely that most of the remaining $\mathrm{Npas}^{+} / \mathrm{FoxP}^{-} / \mathrm{PV}^{-}$pallidostriatal
$5 B)$. Arkypallidal neurons innervate striatum but not STN (Fig. 5B).

\section{Identified prototypic GPe neurons and arkypallidal neurons have different firing rates and patterns in dopamine-intact rats in vivo}

Prototypic GPe neurons and arkypallidal neurons exhibit distinct firing rates and patterns in the dopamine-depleted parkinsonian brain in vivo (Mallet et al., 2012), but whether this holds true for these cell types in the normal brain is unknown. To address this, we first recorded individual GPe neurons in anesthetized, dopamine-intact rats during two extreme brain states (SWA and spontaneous activation), as evinced in frontal ECoGs (Mallet et al., 2008b). We then juxtacellularly labeled each neuron with neurobiotin for post hoc testing of molecular phenotype (Mallet et al., 2012). Given that prototypic neurons coexpress Nkx2-1 and Lhx6 (with most additionally expressing PV), and that arkypallidal neurons express FoxP2 (Fig. 5), we used a series of immunoreactions for these and other markers to assign a cell-type identity to each GPe neuron that was recorded in vivo and labeled with neurobiotin.

When the sampled population was considered as a whole, the spike firing of GPe neurons during SWA was extremely variable; some neurons exhibited vigorous, regular discharges (mean firing rate of up to $\sim 50 \mathrm{spk} / \mathrm{s}$ ), whereas other neurons had sporadic activity and were almost quiescent (mean rate of $\sim 0.02 \mathrm{spk} / \mathrm{s}$ ) (Fig. $6 A-C)$. The firing rates and the fluctuations in firing regularity or "jitter" (as estimated from the SD of ISIs) of recorded GPe neurons varied almost continuously across the sampled population, and the relationship between average firing rate and $\mathrm{SD}_{\text {ISI }}$ was well described by a hyperbolic function (Fig. $6 D$ ), in line with a previous study in isoflurane-anesthetized rats (Deister et al., 2013). However, and most importantly, we found that GPe neurons with different molecular signatures tended to distribute within distinct and restricted aspects of this firing rate/pattern 
A

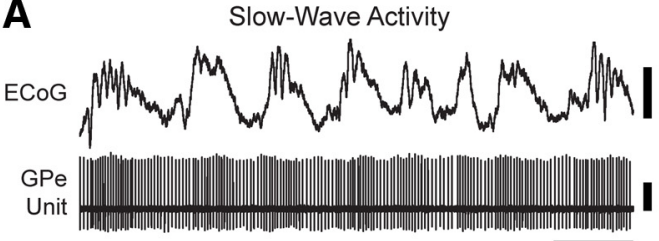

Cortical Activation GPe
Unit U.

B Slow-Wave Activity

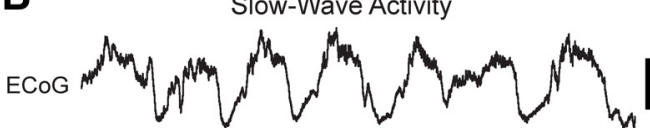

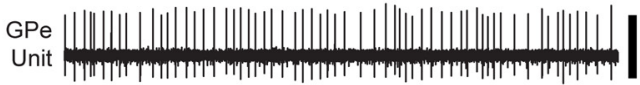

Cortical Activation
ECoG

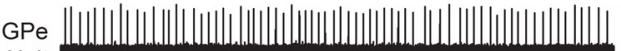

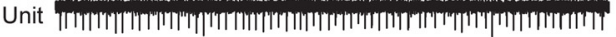

C

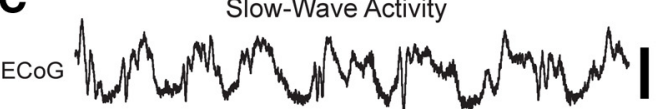

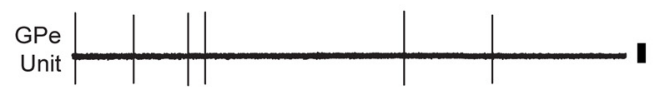

Cortical Activation
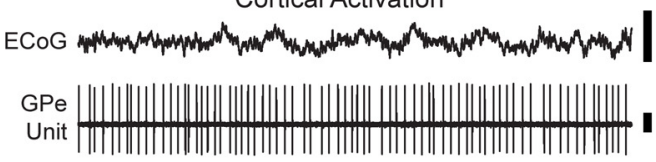

\section{H}

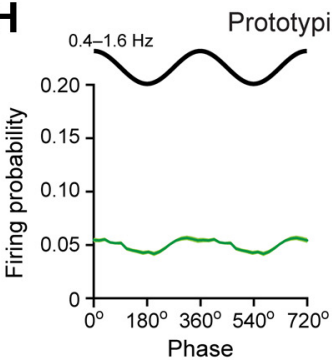

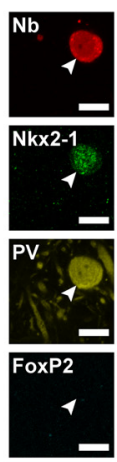
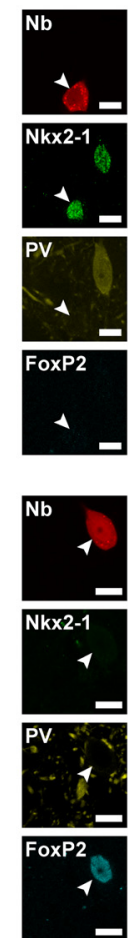

D

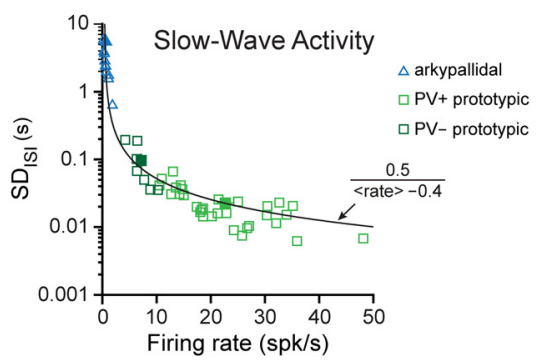

\section{E}

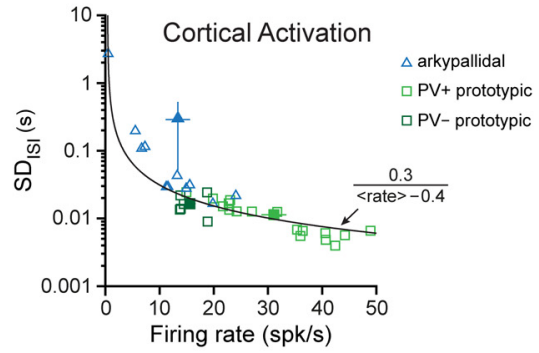

$\mathbf{F}$
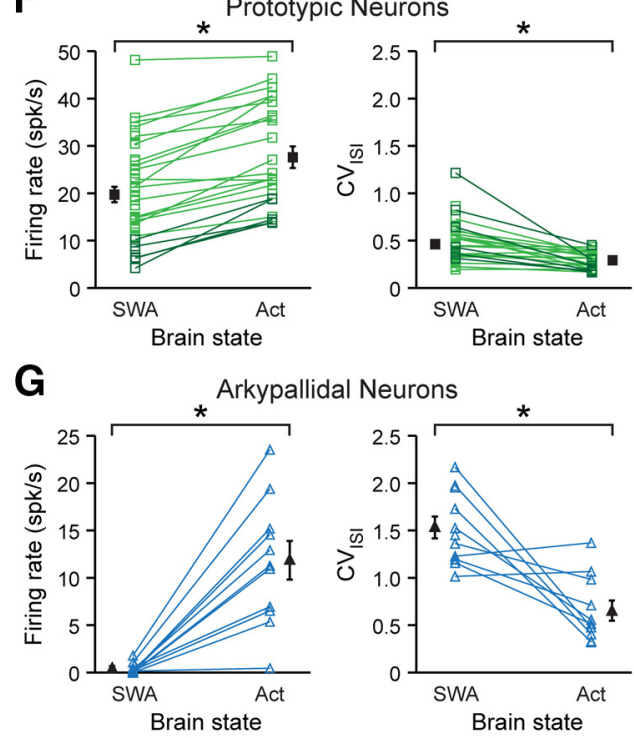

I

Figure 6. The in vivo firing of molecularly identified prototypic neurons and arkypallidal neurons is distinct in dopamine-intact rats. $A$, Typical single-unit activity of a $\mathrm{PV}^{+}$prototypic $\mathrm{GPe}$ neuron recorded in a dopamine-intact adult rat. The same unit was recorded during cortical SWA and cortical "activation," as defined in ECOG. Regardless of brain state, the unit fired at relatively high rates and regular patterns. After recording, the same unit was juxtacellularly labeled with neurobiotin ( $\mathrm{Nb}$ ) and then tested for its expression of various molecular markers (insets). This neuron (arrowhead) coexpressed Nkx2-1 and PV, but not FoxP2, thus identifying it as prototypic. B, Typical activity of a $\mathrm{PV}^{-}$prototypic GPeneuron. C, Typical single-unit activity of an arkypallidal neuron recorded in a dopamine-intact adult rat. The unitfired at relatively low rates and with comparatively irregular patterns during SWA but increased its firing rate and regularity during cortical activation. This GPe neuron expressed FoxP2, but not Nkx2-1 or PV, thus identifying it as arkypallidal. $\boldsymbol{D}$, Plot of the $S D_{|S|}$ against mean firing rate for each GPeneuron recorded during SWA. Open symbols represent individual neurons. Solid symbols represent mean \pm SEM for each cell type. Black line indicates hyperbolic function fitted to the data. Arkypallidal neurons and prototypic neurons tended to distribute within distinct and restricted aspects of the firing rate/pattern continuum. $\boldsymbol{E}$, Same as in $\boldsymbol{D}$ but for GPe neurons recorded during cortical activation. $\boldsymbol{F}$, Plots of the firing rates and $\mathrm{CV}_{\mathrm{ISI}}$ for each prototypic neuron recorded during SWA and/or activation (Act). Open symbols represent individual neurons. Solid symbols represent mean \pm SEM for this cell type. $\mathbf{G}$, Same as in $\boldsymbol{F}$ but for arkypallidal neurons. $\boldsymbol{H}$, Linear phase histograms (left) and circular plots (right) for all spikes of all prototypic neurons with firing that was significantly phase-locked to cortical slow oscillations $(0.4-1.6 \mathrm{~Hz})$. For clarity, two cortical slow oscillation cycles are shown in linear histograms, with data therein represented as mean \pm SEM. In circular plots, vectors of the preferred firing of individual neurons are shown as lines radiating from the center. Greater vector lengths indicate lower variance in the distribution around the mean phase angle (i.e., tighter locking). Each circle on the plot perimeter represents the preferred phase (i.e., mean phase of all the spikes) of an individual neuron. The population vector length and mean angle for the preferred phases of all prototypic neurons is shown as a thick black line. On average, the firing of prototypic neurons was weakly phase-locked to the peaks of cortical slow oscillations. I, Same as in $\boldsymbol{H}$ but for arkypallidal neurons. On average, the firing of arkypallidal neurons was strongly phase-locked to oscillation peaks. Vertical calibration bars: $\boldsymbol{A}-\boldsymbol{C}, 0.5 \mathrm{mV}(\mathrm{ECOG}), 1 \mathrm{mV}$ (units). Horizontal calibration bars: $\boldsymbol{A}-\boldsymbol{C}, 1 \mathrm{~s} .{ }^{*} p<0.05$ (Mann-Whitney U test). Scale bars $A-C$, insets, $10 \mu \mathrm{m}$. 
continuum (Fig. 6D). Indeed, all identified Nkx2-1 ${ }^{+} / \mathrm{PV}^{+} \mathrm{GPe}$ neurons $(n=31)$ fired at relatively high mean rates $(>10 \mathrm{spk} / \mathrm{s})$ and with comparatively regular patterns $\left(\mathrm{CV}_{\mathrm{ISI}}<0.9\right)$ during SWA (Fig. $6 A, D, F)$. Identified Nkx2-1 ${ }^{+} / \mathrm{PV}^{-}$neurons $(n=7)$ fired at significantly lower rates than $\mathrm{PV}^{+}$GPe neurons, although their firing regularities were similar $(p<0.001$ and $p=0.228$, respectively, Mann-Whitney tests; Fig. $6 B, D, F)$. As expected from our population-level cell counts (Figs. 1234-5), all recorded $\mathrm{Nkx} 2-1^{+}$neurons were also FoxP2 ${ }^{-}$, thus further ascribing the prototypic cell-type identity to them. In general, the spike firing of prototypic GPe neurons $(n=38)$ was not strongly timed with the ongoing cortical slow oscillations $(\sim 1 \mathrm{~Hz})$ that prevailed during SWA (Fig. $6 A, B$ ), but there were a few exceptions (see below). In contrast to the firing of prototypic GPe neurons, identified FoxP2 ${ }^{+}$GPe neurons $(n=13)$ fired at low mean rates $(<2 \mathrm{spk} / \mathrm{s})$ and in a less regular manner $\left(\mathrm{CV}_{\text {ISI }}>1\right)$ during SWA (Fig. $6 C, D, G)$. Five of these FoxP2 ${ }^{+}$neurons were tested for coexpression of PPE and, as could be predicted from our population cell counts, all five were also PPE ${ }^{+}$. All 13 FoxP2 ${ }^{+}$neurons tested negative for expression of PV. Eight of these FoxP2 ${ }^{+} / \mathrm{PV}^{-}$neurons were additionally tested for $\mathrm{Nkx} 2-1$ expression; none of these neurons were $\mathrm{Nkx} 2-1^{+}$, thus further ascribing an arkypallidal identity to the FoxP2 ${ }^{+}$GPe neurons recorded here. These arkypallidal neurons typically fired single spikes, or small groups of spikes, in time with the peaks of cortical slow oscillations (Fig. 6C).

Defining whether and how the spike firing of BG neurons is temporally related to the stereotyped cortical slow oscillation have utility for elucidating the potential contributions of different sets of inputs to BG activity (Magill et al., 2001; Walters et al., 2007). Although the power of slow oscillations in frontal ECoGs recorded with arkypallidal neurons or prototypic neurons was similar (Mann-Whitney test on ECoG power in $0.4-1.6 \mathrm{~Hz}$ band), the activity of arkypallidal neurons appeared more tightly coupled to the ongoing slow oscillations (Fig. 6A-C). To further examine the temporal relationship between activity in GPe and cortex during SWA, we used the Hilbert transform to analyze the instantaneous phase of the spiking of arkypallidal and prototypic neurons with respect to cortical slow oscillations (Nakamura et al., 2014). To qualify for these and related circular statistical analyses, a GPe neuron had to fire $\geq 40$ spikes during the recording, a sampling criterion that helped to ensure accurate determination of circular means, and the significance of any phase-locked firing (Nakamura et al., 2014). This meant that 4 arkypallidal neurons with very low firing rates (means of $<0.1 \mathrm{spk} / \mathrm{s}$ ) were excluded from these analyses. The spikes of all qualifying arkypallidal neurons $(n=9)$ were significantly locked to the slow oscillations (Rayleigh's Uniformity Tests). A smaller proportion of $\mathrm{PV}^{+}$pro- $^{-}$ totypic neurons ( $n=26$ of 31 qualifying neurons) showed significant phase locking, but all $\mathrm{PV}^{-}$prototypic neurons $(n=7)$ were significantly locked. Other than this difference between $\mathrm{PV}^{+}$and $\mathrm{PV}^{-}$prototypic neurons, their firing with respect to slow oscillations was similar, and so, the two groups of prototypic neurons were pooled for further analysis. When the spike train of each significantly phase-locked arkypallidal neuron $(n=9)$ and prototypic neuron $(n=34)$ was analyzed, both types of GPe neuron preferentially fired around the peak of cortical slow oscillations, defined as a phase angle of between $>270^{\circ}$ and $<90^{\circ}$ (Fig. $6 \mathrm{H}, \mathrm{I}$ ). However, compared with prototypic GPe neurons, the peak of activity of arkypallidal neurons was larger in the phase histogram (Fig. $6 H, I$ ), giving a first indication of tighter phase locking as a population. Circular plots of the preferred phases of these individual phase-locked arkypallidal and prototypic neurons con- firmed the strong tendency of the former cell type to fire around the peaks of the slow oscillations (Fig. $6 H, I$ ). Indeed, although all arkypallidal neurons preferentially fired in time with peaks (Fig. $6 I$ ), not all prototypic neurons preferentially fired likewise; $20 \%$ of prototypic neurons fired more in time with the troughs of the slow oscillations (Fig. $6 H$ ). Although the mean angles of the preferred phases (i.e., the circular means) were similar for the two cell types (arkypallidal neurons, $343.4 \pm 12.4^{\circ}$; prototypic neurons, $348.4 \pm 10.2^{\circ}$; Watson-Williams $F$ test), the vector lengths of arkypallidal neurons were significantly greater than those of prototypic neurons ( $p<0.001$, Mann-Whitney test), thus confirming that the preferred firing of individual arkypallidal neurons was comparatively more concentrated (i.e., more tightly locked).

Most prototypic neurons (24 of 38 ) were subsequently recorded during periods of spontaneous cortical activation, as evinced by a loss of ECoG slow oscillations (Fig. 6A, B). On average, the firing rate and regularity of prototypic neurons during cortical activation were significantly higher than those during SWA ( $p=0.007$ and $p<0.001$, respectively, Mann-Whitney tests), with almost all of the tested neurons (23 of 24) responding with modest increases in their firing during transition from SWA to an activated brain state (Fig. $6 F$ ). Most arkypallidal neurons (11 of 13) were also recorded during spontaneous cortical activation (Fig. 6C). Both the average firing rate and regularity of arkypallidal neurons during activation were significantly higher than those during SWA ( $p<0.001$ and $p<0.001$, respectively, Mann-Whitney tests), with almost all tested neurons (10 of 11) responding with pronounced increases in their firing rates during brain state transition (Fig. 6G). On average, arkypallidal neurons fired at significantly lower rates and regularities than prototypic GPe neurons during both SWA and activation (all $p<0.001$, Mann-Whitney tests). However, during activation, there was a marked overlap in the firing properties of the two cell types (Fig. $6 E)$, reiterating the necessity of controlling for brain state as well as the importance of unequivocally identifying the cell type of neurons recorded in GPe in vivo.

Together, these data show that, in the dopamine-intact brain in vivo, molecularly identified prototypic GPe neurons and arkypallidal neurons exhibit largely distinct firing rates and regularities (regardless of brain state), as well as dissimilar levels of temporal coupling with ongoing cortical slow oscillations during SWA.

\section{The autonomous firing and intrinsic membrane properties of identified prototypic GPe neurons and arkypallidal neurons are distinct}

The distinct firing rates/patterns of prototypic and arkypallidal neurons in the dopamine-intact brain in vivo could arise, at least in part, because these cell types have different intrinsic membrane properties. To address this possibility, we recorded the autonomous and driven firing of individual, molecularly identified GPe neurons in brain slices acutely prepared from juvenile (P20-P35) dopamine-intact rats. We first tested whether some definitive aspects of the molecular profiles of neurons in adult rat GPe held true for neurons in juvenile rat GPe. On average, $\mathrm{Nkx2-1}{ }^{+}$neurons and $\mathrm{PV}^{+}$neurons constituted, respectively, just over twothirds (69\%) and one-half (52\%) of all GPe neurons. Most (73\%) Nkx2-1 ${ }^{+}$neurons coexpressed PV, whereas nearly all $(99 \%)$ $\mathrm{PV}^{+}$neurons coexpressed Nkx2-1. On average, FoxP2 ${ }^{+}$neurons comprised just over one-fourth (28\%) of all GPe neurons. There was a high degree of overlap in the expression of FoxP2 and PPE; $94 \%$ of FoxP2 ${ }^{+}$neurons coexpressed PPE, and $>99 \%$ of $\mathrm{PPE}^{+}$ 
neurons coexpressed FoxP2. Importantly, coexpression of Nkx2-1 and FoxP2, or coexpression of PV and FoxP2, by individual neurons in juvenile GPe was very rare $\left(<1 \%\right.$ of $\mathrm{Nkx} 2-1^{+}$or $\mathrm{PV}^{+}$neurons coexpressed FoxP2 and vice versa). These cell counts from juvenile rat GPe are in good agreement with those from adult rat GPe (Fig. 5A) and suggest that the definitive molecular architecture of adult rat GPe neurons is largely established by the fourth postnatal week. Moreover, these findings confirm that PV expression and FoxP2 expression mark prototypic neurons and arkypallidal neurons, respectively, in the juvenile rat GPe. Thus, after electrophysiological recording in vitro, each GPe neuron was filled with biocytin, recovered, and tested for expression of PV and FoxP2 immunoreactivities.

The autonomous spike firing of synaptically isolated GPe neurons recorded in a perforated-patch configuration was highly variable; some neurons consistently engaged in robust, regular activity (mean firing rate of up to $\sim 55 \mathrm{spk} / \mathrm{s}$ ), whereas other neurons fired only infrequently $(\sim 1 \mathrm{spk} / \mathrm{s})$ (Fig. $7 A-D)$. The autonomous firing rates and jitter of recorded GPe neurons varied almost continuously across the sampled population, and the relationship between average firing rate and $\mathrm{SD}_{\mathrm{ISI}}$ was well described by a hyperbolic function (Fig. 7D), similar to that reported in a recent in vitro study (Deister et al., 2013). Thus, in these respects, the diverse GPe neuron firing that we observed in vivo was recapitulated in vitro. In further keeping with our in vivo data, we observed that GPe neurons with different molecular signatures tended to distribute within distinct aspects of this autonomous firing rate/pattern continuum (Fig. 7D). All identified $\mathrm{PV}^{+}$prototypic neurons tested $(n=14)$ were autonomously active, with most (79\%) firing spikes at relatively high mean rates $(>10 \mathrm{spk} / \mathrm{s})$ and regularities $\left(\mathrm{CV}_{\mathrm{ISI}}<0.13\right)$ (Fig. $\left.7 A, D\right)$. Identified FoxP2 ${ }^{+}$arkypallidal neurons $(n=18)$ were also autonomously active, but at comparatively low mean rates (only one neuron fired at $>10 \mathrm{spks} / \mathrm{s}$ ) and regularities (only $44 \%$ of neurons had $\mathrm{CV}_{\text {ISI }}<0.13$ ) (Fig. $7 C, D$ ). A third, smaller group of $\mathrm{PV}^{-} / \mathrm{FoxP}^{-}$GPe neurons $\left(n=5\right.$; presumably, $\mathrm{PV}^{-}$prototypic neurons) also displayed autonomous activity, but with firing rates and regularities that were often in between those of $\mathrm{PV}^{+}$ and FoxP2 ${ }^{+}$neurons (Fig. 7 B,D). On average, arkypallidal neurons fired at significantly lower rates and regularities than $\mathrm{PV}^{+}$ prototypic neurons $(p<0.001$ and 0.026 , respectively, KruskalWallis ANOVAs; Fig. 7E). The same held true when all prototypic neurons were pooled together $(p<0.001$ and $p<0.001$, MannWhitney tests). These observations again mirror our in vivo data. Input resistances, and action potential waveforms and thresholds, were similar across these groups of GPe neurons (Table 1). An analysis of autonomous firing showed that the average magnitude of the afterhyperpolarization potential (AHP) of the action potentials fired by arkypallidal neurons was almost twice that of prototypic neurons (Table 1). However, when the firing of arkypallidal and prototypic neurons was driven with current injections to an equivalent rate $(\sim 40 \mathrm{spk} / \mathrm{s})$, the AHP magnitudes of the cell types were similar (Table 1).

Somatic injections of depolarizing current pulses revealed that the "driven" firing of arkypallidal neurons was not as vigorous as that of prototypic neurons (Fig. $7 A-C$; Table 1). In line with this, the F-I response curves of these cell types was markedly different (Fig. $7 F$ ). More specifically, the driven firing rates of arkypallidal neurons were significantly slower across a range of current pulses compared with those of prototypic neurons (Fig. $7 F$ ), suggesting that arkypallidal neurons are comparatively less responsive to excitation. Upon somatic injections of hyperpolarizing current pulses, both prototypic and arkypallidal neurons exhibited a membrane potential "sag" (Fig. $7 A-C)$. When prototypic and arkypallidal neurons were hyperpolarized to equivalent peak voltage deflections (to $\sim-100 \mathrm{mV}$ [Fig. $7 A-C$ ], or to $\sim-90,-80$, or $-70 \mathrm{mV}$ ), their sag amplitudes were similar (Kruskal-Wallis ANOVAs). These data suggest that the subunit composition, density, and/or distribution of hyperpolarizationactivated cyclic nucleotide-gated cation (HCN) channels along the somatodendritic membranes of the two cell types are also similar (Biel et al., 2009), although voltage-clamp recordings would be needed to resolve this in a more definitive manner. Compared with prototypic neurons, a smaller proportion of arkypallidal neurons had significant "rebound" firing at the break of hyperpolarizing currents pulses, and the mean rates of arkypallidal neuron rebound firing were also lower (Fig. 7A-C; Table 1). Together, these results support the conclusion that prototypic neurons and arkypallidal neurons differ in their autonomous firing and some other active membrane properties.

\section{Prototypic and arkypallidal neurons exhibit different propensities to engage in autonomous "rate wandering"}

Individual GPe neurons (of unknown cell type or types) can display a large degree of heterogeneity in the rate and regularity of their autonomous firing (Deister et al., 2013). Indeed, longduration recordings in vitro have revealed that the firing of individual neurons can slowly (over a few minutes), spontaneously, and independently fluctuate or "wander," such that one cell can eventually visit many of the rates and patterns seen in the population activity continuum (Deister et al., 2013). These data suggest that caution must be exercised when classifying GPe neurons on the basis of electrophysiological parameters alone, and further raise the possibility that the distinct autonomous firing of prototypic and arkypallidal neurons might not hold true across long sampling periods. To address this issue and test whether rate wandering is a feature common to both GPe cell types, we conducted long-duration (15-30 min) cell-attached recordings of molecularly identified neurons (Fig. 8). On average, the firing rates of prototypic and arkypallidal neurons in cell-attached recordings were, respectively, similar to those of these cell types recorded for shorter durations in a perforated-patch configuration (Mann-Whitney tests). However, the prolonged cellattached recordings suggested that these cell types have different tendencies to engage in slow activity fluctuations. We observed that the autonomous firing of individual $\mathrm{PV}^{+}$prototypic neurons $(n=8)$ did not fluctuate much over these long recording periods (Fig. $8 A$ ), and the ISI histograms of these neurons were typically symmetrical (Fig. $8 B$ ). In comparison, the firing of FoxP2 ${ }^{+}$arkypallidal neurons $(n=6)$ and $\mathrm{PV}^{-} / \mathrm{FoxP}^{-}$prototypic neurons $(n=4)$ wandered to greater extents over time, and their ISI histograms were positively skewed and leptokurtic (Fig. $8 C-F$ ). Although most $\mathrm{PV}^{+}$prototypic neurons (6 of 8 ) had ISI distributions with small values of kurtosis and skewness (i.e., values between -2 and 2), neither arkypallidal neurons nor $\mathrm{PV}^{-}$ prototypic neurons had ISI distributions in this range (Fig. 8G). These data indicate that the overall consistency of autonomous firing rate/pattern is dependent on GPe cell type. To further examine the propensities of different cell types to engage in rate wandering, we partitioned the long-duration recording of each neuron into groups of 100 adjacent spikes and plotted the $\mathrm{SD}_{\mathrm{ISI}}$ within each group against the corresponding mean firing rate (Fig. $8 H, I$ ). This analysis showed that the firing of individual arkypallidal neurons and $\mathrm{PV}^{-}$prototypic neurons wandered to a greater extent than that of individual $\mathrm{PV}^{+}$prototypic neurons, but also that individual neurons visited only a limited aspect of 

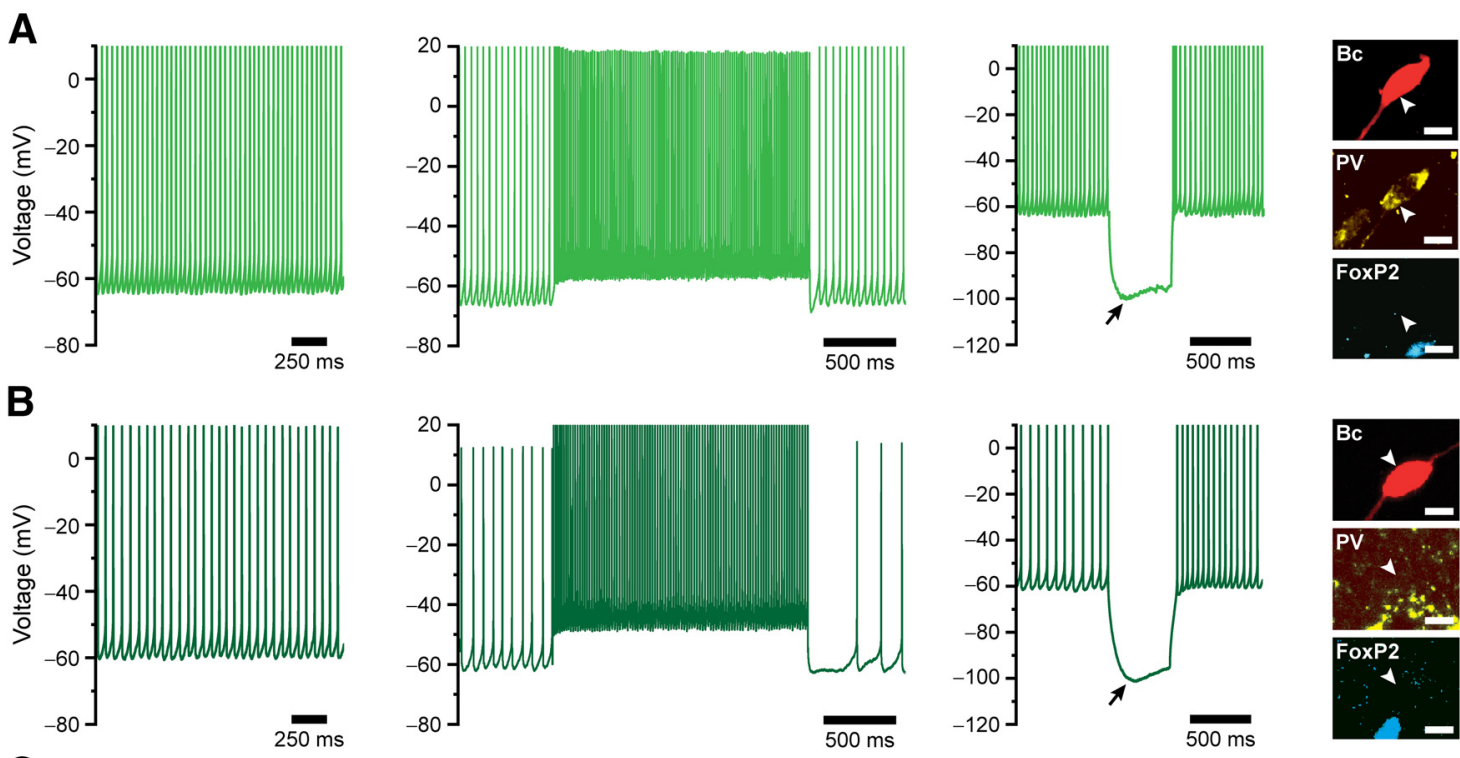

C
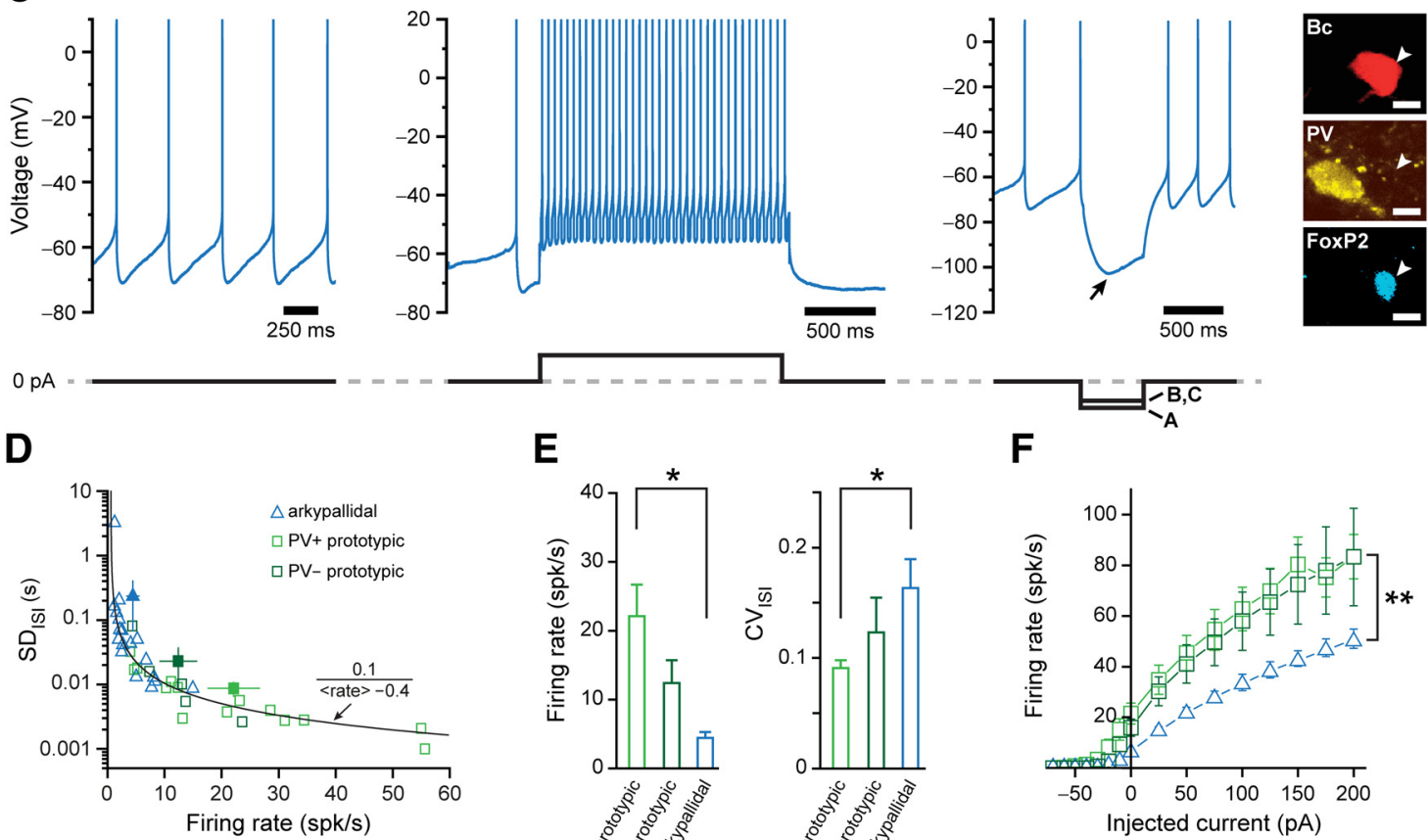

E

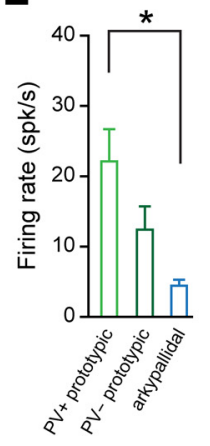

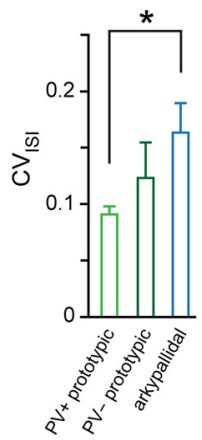

$\mathbf{F}$

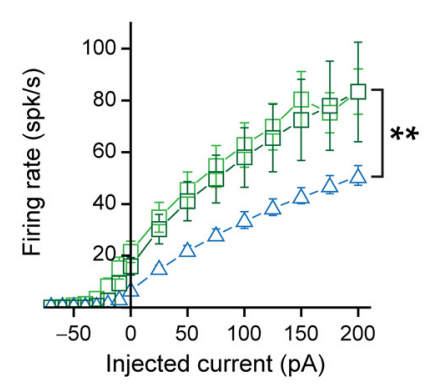

Figure 7. Autonomous firing and intrinsic membrane properties of molecularly identified prototypic neurons and arkypallidal neurons are distinct in vitro. $A$, Typical activity of a PV ${ }^{+}$prototypic GPe neuron recorded in a perforated-patch configuration in a brain slice from a juvenile dopamine-intact rat. Autonomous firing ( $0 \mathrm{pA}$ current injection), driven firing (100 pA injection for $2 \mathrm{~s}$ ), and response to hyperpolarizing current pulse ( $-100 \mathrm{pA}$ or $-80 \mathrm{pA}$ for $500 \mathrm{~ms}$; eliciting peak voltage deflections of $\sim-100 \mathrm{mV}$ and a subsequent "sag" [arrows]) are shown from left to right. After perforated-patch recording, the same neuron was repatched and filled with biocytin (BC), and then tested for its expression of molecular markers (insets). This neuron (arrowhead) expressed PV, but not FoxP2, thus identifying it as prototypic. $\boldsymbol{B}$, Typical activity of a $\mathrm{PV}^{-}$prototypic GPe neuron. $\boldsymbol{C}$, Typical activity of an arkypallidal neuron. The neuron fired at relatively low rates. This $\mathrm{GPe}$ neuron expressed FoxP2, but not PV, thus identifying it as arkypallidal. $\boldsymbol{D}$, Plot of the $\mathrm{SD}_{\mid \mathrm{SI}}$ against mean firing rate for each GPe neuron recorded in the perforated-patch configuration. Open symbols represent individual neurons. Solid symbols represent mean \pm SEM for each cell type. Black line indicates hyperbolic function fitted to the data. Arkypallidal neurons and prototypic neurons tended

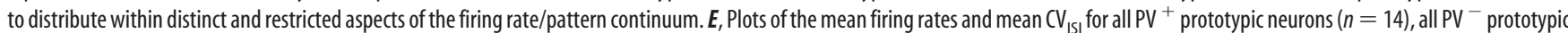
neurons $(n=5)$, and all arkypallidal neurons $(n=18)$. On average, prototypic neurons fire at higher rates and regularities than arkypallidal neurons. ${ }^{*} p<0.05$ (Kruskal-Wallis ANOVA, followed by Dunn's test). $\boldsymbol{F}$, Relationship between mean firing rate and injected current (i.e., the F-I response curves). For depolarizing pulses, arkypallidal neuron firing was characterized by a significantly slower rate of growth compared with prototypic neurons. ${ }^{*}$ Significantly different firing rates with all current injections ranging from $-10 \mathrm{pA}$ to $200 \mathrm{pA}(p<0.05$, Kruskal-Wallis ANOVAs, followed by Dunn's tests). $\boldsymbol{E}, \boldsymbol{F}$, Data are mean \pm SEM. Scale bars $\boldsymbol{A}-\boldsymbol{C}$, insets, $10 \mu \mathrm{m}$.

the population activity continuum (Fig. $8 H$ ). When all neurons recorded in a cell-attached configuration were considered, it was evident that, although arkypallidal neurons engaged in modest activity fluctuations over time, their firing rates and regularities did not achieve those typical of $\mathrm{PV}^{+}$prototypic neurons (Fig. $8 I$ ).
In summary, these results show that the degree to which individual GPe neurons engage in rate wandering is cell-type dependent and further suggest that the different autonomous firing of prototypic and arkypallidal neurons is unlikely to arise from undersampling of slow activity fluctuations. Similarly, these in vitro results 
Table 1. Electrophysiological properties of prototypic and arkypallidal neurons in vitro ${ }^{a}$

\begin{tabular}{|c|c|c|c|c|}
\hline \multirow[b]{2}{*}{ Marker expression } & \multicolumn{2}{|l|}{ Prototypic } & \multicolumn{2}{|l|}{ Arkypallidal* $^{*}$} \\
\hline & $\mathrm{PV}^{+}$ & $\mathrm{PV}^{-} / \mathrm{FoxP2}^{-}$ & $\mathrm{FoxP}^{+}$ & $p$ \\
\hline No. of neurons tested & 14 & 5 & 18 & - \\
\hline APth (mV) & $-56.6 \pm 1.8$ & $-49.9 \pm 2.8$ & $-55.0 \pm 1.8$ & 0.208 \\
\hline APhw (ms) & $0.78 \pm 0.07$ & $0.73 \pm 0.13$ & $0.98 \pm 0.16$ & 0.394 \\
\hline $\operatorname{AHP}(m V)$ & $-67.3 \pm 2.2$ & $-66.7 \pm 1.8^{* *}$ & $-73.3 \pm 1.8^{* *}$ & 0.044 \\
\hline AHP magnitude (APth - AHP) at autonomous firing rates & $10.6 \pm 1.2^{* *}$ & $16.8 \pm 2.6$ & $18.2 \pm 1.2^{* *}$ & 0.001 \\
\hline Driven firing adaptation index & $0.64 \pm 0.03$ & $0.69 \pm 0.11$ & $0.78 \pm 0.04$ & 0.075 \\
\hline No. of neurons with rebound & $12 / 13(92 \%)$ & $4 / 5(80 \%)$ & $9 / 16(56 \%)$ & - \\
\hline Rebound firing rate (spk/s) & $46.6 \pm 13.5^{* *}$ & $21.7 \pm 5.1$ & $9.9 \pm 3.2^{* *}$ & 0.012 \\
\hline
\end{tabular}

$\bar{a}$ Summary of intrinsic properties of prototypic and arkypallidal neurons recorded in a perforated-patch configuration. Values are given as mean \pm SEM. APth, Action potential threshold; APhw, action potential half-width. Driven firing rate and adaptation were assessed with a $2 \mathrm{~s}$ current pulse of $100 \mathrm{pA}$. Mean rebound firing rate was assessed after a $500 \mathrm{~ms}$ current pulse of $-150 \mathrm{pA}$; one PV ${ }^{+}$prototypic neuron and two arkypallidal neurons could not be tested for rebound firing because of premature entry into a whole-cell configuration.

*Reported for Kruskal-Wallis ANOVA on Ranks.

**Neuron groups with statistically different values ( $p<0.05$; post hoc Dunn's test). When all prototypic neurons were pooled ( $n=19$ ) and their intrinsic properties were compared with those of arkypallidal neurons (Mann-Whitney tests), all parameters, except input resistance, APth, APhw, and AHP magnitude at $\sim 40 \mathrm{spk} / \mathrm{s}$, were significantly different.

A

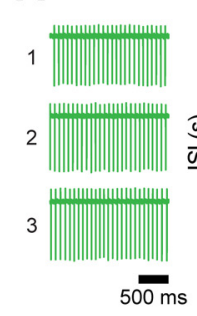

C

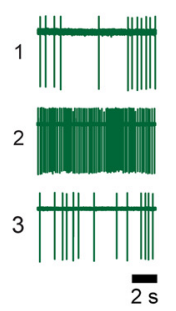

E

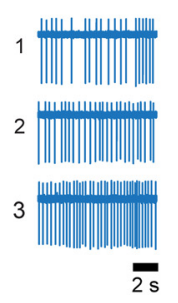

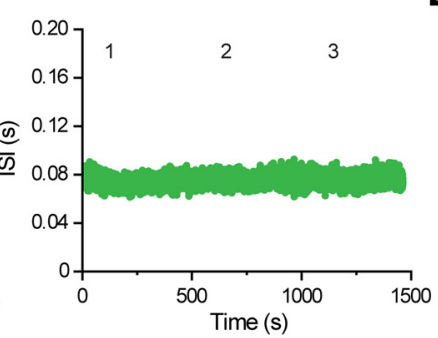
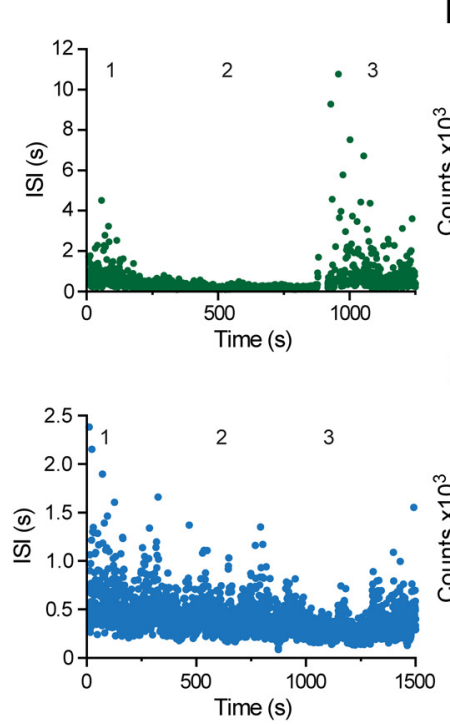

B

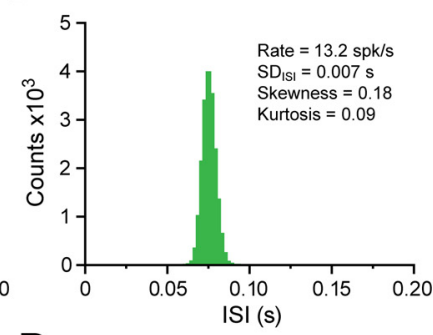

D
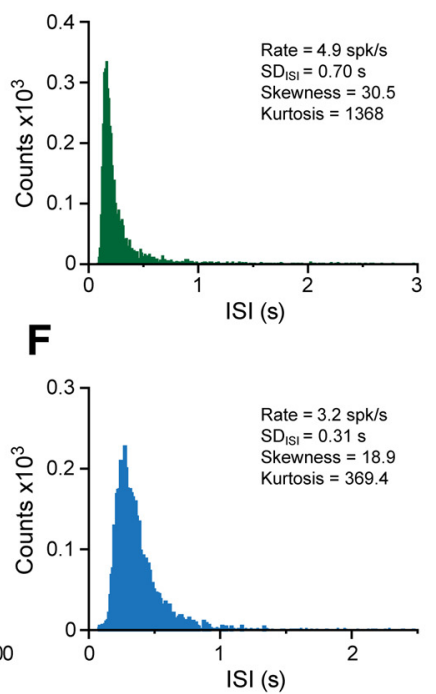

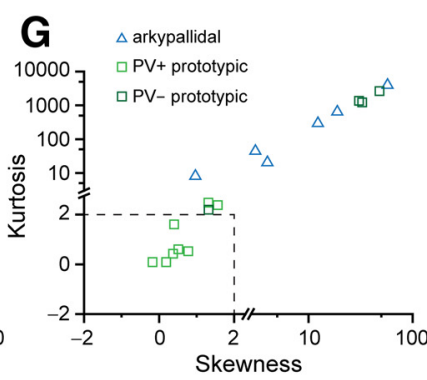

$\mathbf{H}$
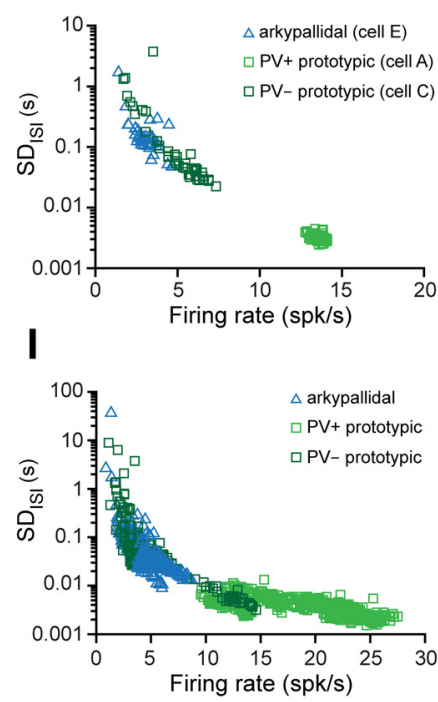

Figure 8. Prototypic neurons and arkypallidal neurons engage in autonomous "rate wandering" to different extents. $A$, Typical examples of the spike firing and ISIs of an identified $\mathrm{PV}^{+}$prototypic GPe neuron that was continuously recorded for $25 \mathrm{~min}$ in a cell-attached configuration. Raw traces of firing (left) were taken at time points 1,2 , and 3 indicated on the ISI plot (right). $\boldsymbol{B}, \mathrm{ISI}$ histogram for the same PV ${ }^{+}$prototypic neuron; its mean firing rate, the $\mathrm{SD}_{|S|}$, and the skewness and kurtosis values of its ISI distribution are also indicated. The activity of this neuron did not fluctuate or wander substantially over time, as reflected by its symmetrical ISI distribution and relatively low values of $S_{|S| l}$, kurtosis, and skewness. $C, D$, Typical examples of the spike firing and ISIs of an identified PV ${ }^{-}$prototypic neuron. $\boldsymbol{E}, \boldsymbol{F}$, Typical example of the spike firing and ISIs of an identified arkypallidal neuron. The autonomous firing of the $\mathrm{PV}^{-}$prototypic neuron and the arkypallidal neuron wandered over time, as reflected by their positively skewed ISI distributions and their relatively high values of $S_{\mathrm{ISI}}$ kurtosis, and skewness. G, Plot of kurtosis and skewness values for each prototypic neuron $\left(n=8 \mathrm{PV}^{+} ; n=4 \mathrm{PV}^{-}\right)$and arkypallidal neuron $(n=6)$ recorded for $\geq 15$ min in a cell-attached configuration. The kurtosis and skewness values of most PV ${ }^{+}$prototypic neurons were small and lay between -2 and 2 (dashed lines). For clarity, log scales are used after the axis breaks. $\boldsymbol{H}$, Plot of $S D_{|S|}$ against firing rate for the three example neurons shown in $\boldsymbol{A}, \boldsymbol{C}$, and $\boldsymbol{E}$. Each symbol represents the $S D_{|S|}$ and mean firing rate calculated for a group of 100 adjacent spikes fired by a single neuron. During rate wandering, individual neurons visited only a limited aspect of the activity continuum. $I$, Plot of $S D_{I S I}$ against firing rate for all recorded neurons, with each symbol representing values for a group of 100 adjacent spikes of a single neuron. Even with cell-type-dependent rate wandering, arkypallidal neurons and PV ${ }^{+}$ prototypic neurons tended to distribute within distinct and restricted aspects of the population activity continuum. Bin sizes of histograms: $\boldsymbol{B}, 2 \mathrm{~ms} ; \boldsymbol{D}, 10 \mathrm{~ms} ; \boldsymbol{F}, 10 \mathrm{~ms}$. 
indicate that the different firing rates and patterns of these cell types in vivo cannot be readily explained by intrinsic rate wandering.

\section{Prototypic GPe neurons have larger "persistent" sodium channel currents than arkypallidal neurons}

The pace and precision of the autonomous firing of GPe neurons are critically dependent on a subthreshold (persistent) sodium current mediated by voltage-dependent sodium channels, as well as on other currents mediated by HCN channels, smallconductance calcium-activated potassium (SK) channels (with calcium provided by opening of high voltage-activated calcium $\left(\mathrm{Ca}_{\mathrm{vH}}\right)$ channels), and A-type potassium $\left(\mathrm{K}_{\mathrm{v}} 4\right)$ channels (Chan et al., 2004; Mercer et al., 2007; Deister et al., 2009). Moreover, computer simulations (Günay et al., 2008) suggest that the marked variability in the intrinsic membrane properties of GPe neurons, as defined here and reported previously (Nambu and Llinaś, 1994; Cooper and Stanford, 2000; Günay et al., 2008; Bugaysen et al., 2010; Chuhma et al., 2011; Miguelez et al., 2012; Deister et al., 2013), arises through neurons expressing different densities of the membrane conductances mediated by these and other types of ion channels. We next tested whether the distinct autonomous firing of prototypic and arkypallidal neurons was accompanied by cell-type-dependent differences in their persistent sodium channel currents $\left(\mathrm{I}_{\mathrm{NaP}}\right)$. Using a whole-cell voltageclamp recording configuration, we measured currents evoked in identified GPe neurons by somatic injection of slowly depolarizing ramps of voltage command (Fig. $9 A, B$ ) in the absence and in the presence of $1 \mu \mathrm{M}$ TTX. Isolation of $\mathrm{I}_{\mathrm{NaP}}$ was achieved by subtracting the currents recorded in the control and TTX-infused conditions (Fig. 9A,B). The peak amplitudes of $\mathrm{I}_{\mathrm{NaP}}$ recorded in $\mathrm{PV}^{+}$prototypic neurons $(n=6)$ were, on average, approximately double those in FoxP2 ${ }^{+}$neurons $(n=4)$, without any difference in channel activation kinetics between the two cell types (Fig. $9 C, D$ ) ( $p=0.014$ and $p=0.257$, respectively, MannWhitney tests). This result suggests that the autonomous firing rates of prototypic neurons are higher than those of arkypallidal neurons because the former cell type expresses higher densities of the voltage-dependent sodium channels that mediate the subthreshold sodium conductance important for GPe neuron pacemaking. The smaller $\mathrm{I}_{\mathrm{NaP}}$ of arkypallidal neurons might also partly explain why this cell type exhibited comparatively weak rebound firing (Günay et al., 2008).

\section{Chronic dopamine loss differentially affects the firing of identified prototypic GPe neurons and arkypallidal neurons in vivo}

A previous study in anesthetized parkinsonian rats predicted that chronic dopamine loss imbalances the activities of prototypic and arkypallidal neurons, such that their normal physiological phenotypes in vivo are teased further apart (Mallet et al., 2012). However, the previous use of spike-firing relationships as the sole classifier of cell type (Mallet et al., 2012) is not optimal because electrophysiological phenotypes in vivo are highly dynamic and can overlap, thus leading to ambiguities. Having defined the in vivo firing properties of prototypic and arkypallidal neurons in the dopamine-intact GPe here, we took the opportunity to test this important prediction in a final comparative analysis that used molecular expression profiles as the cell-type classifier. We thus quantified the extent to which chronic dopamine loss, induced by unilateral 6-OHDA lesions targeted to the midbrain (Mallet et al., 2008a; Mallet et al., 2012), alters the firing properties of molecularly defined arkypallidal and prototypic neurons in vivo. All arkypallidal neurons $(n=9)$ recorded in anesthetized
A
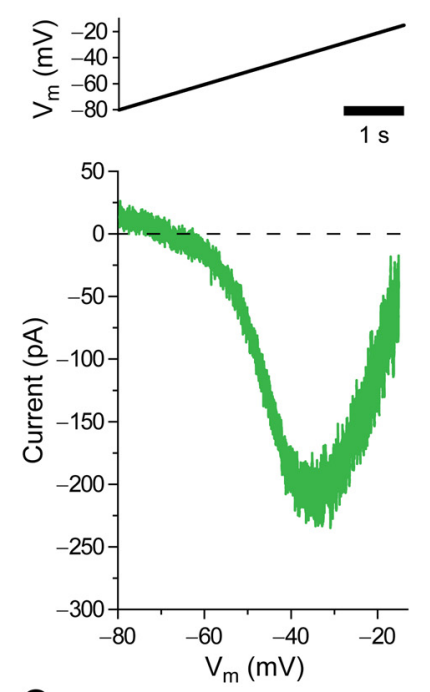

C

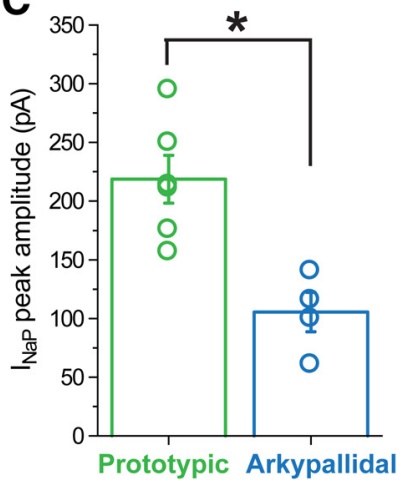

B
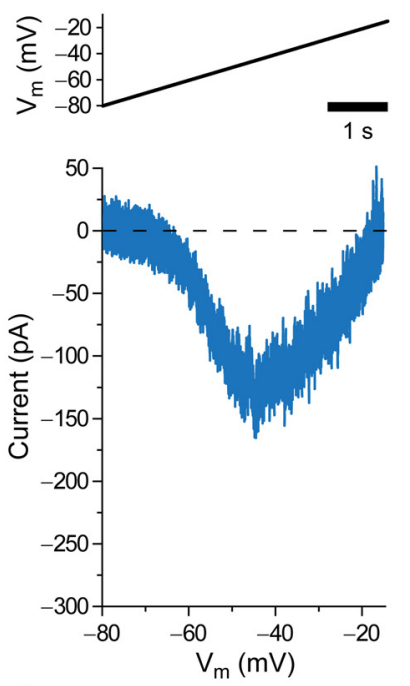

D

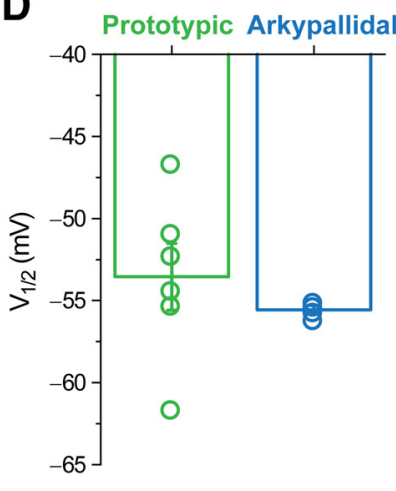

Figure 9. Amplitudes of persistent sodium channel currents in identified prototypic neurons and arkypallidal neurons are different. $\boldsymbol{A}, \boldsymbol{B}$, Representative in vitro recordings of persistent sodium channel currents $\left(\mathrm{I}_{\mathrm{NaP}}\right.$, bottom traces) evoked in a $\mathrm{PV}^{+}$prototypic neuron $(\boldsymbol{A})$ and a FoxP2 ${ }^{+}$arkypallidal neuron $(\boldsymbol{B})$ upon somatic injections of a ramp voltage command (thick black line in upper schematics). $V_{m}$, Membrane voltage. Traces were obtained by subtraction of currents evoked under control and TTX-infused conditions. C, Average peak amplitude of $\mathrm{I}_{\mathrm{NaP}}$ in $\mathrm{PV}^{+}$prototypic neurons (green) was significantly larger than that in $\mathrm{FoxP}^{+}$arkypallidal neurons (blue). $\boldsymbol{D}$, Average half-activation voltages $\left(\mathrm{V}_{1 / 2}\right)$ for $\mathrm{I}_{\mathrm{NaP}}$ in prototypic and arkypallidal neurons were similar. ${ }^{*} p=0.014$ (Mann-Whitney $U$ test). C, D, Error bars indicate mean \pm SEM; circles represent data points for individual neurons superimposed.

6-OHDA-lesioned rats were identified as such by their expression of FoxP2; all of these neurons also expressed PPE but also tested negative for PV expression. Prototypic neurons $(n=30)$ recorded in lesioned rats were initially identified by their lack of FoxP2 expression; 12 of 30 FoxP2 ${ }^{-}$neurons were tested for Nkx2-1 expression and all were $\mathrm{Nkx} 2-1^{+}$.

In 6-OHDA-lesioned rats, the spike firing of GPe neurons during SWA was again extremely variable; some neurons discharged at high rates (mean rate of up to $\sim 40 \mathrm{spk} / \mathrm{s}$ ), whereas other neurons were almost quiescent (mean rate $\sim 0.01 \mathrm{spk} / \mathrm{s}$ ) (Fig. 10A-C). Across the sampled cell population, there was again a firing rate/jitter continuum that was well described by a hyperbolic function (Fig. 10D). However, molecularly defined prototypic and arkypallidal neurons tended to distribute within distinct and restricted aspects of this continuum (Fig. 10D), in line with data from dopamine-intact animals. Thus, all $\mathrm{PV}^{+}$prototypic neurons $(n=25)$ fired at relatively high mean rates $(>8$ spk/s) during SWA (Fig. 10A,D,F). Although $\mathrm{PV}^{-}$prototypic neurons $(n=5)$ fired at significantly lower rates than $\mathrm{PV}^{+}$neu- 
A

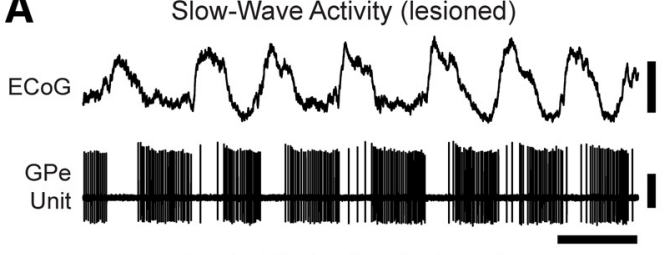

Cortical Activation (lesioned)

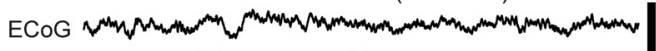

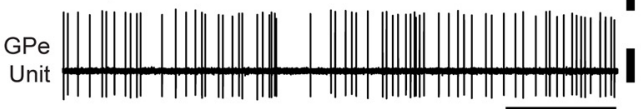

B Slow-Wave Activity (lesioned)

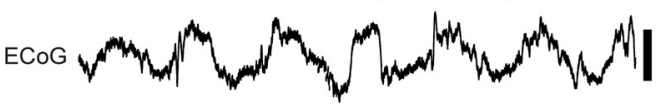

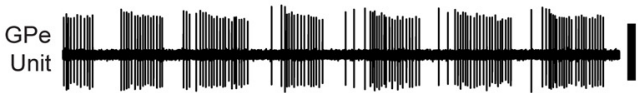

Cortical Activation (lesioned)

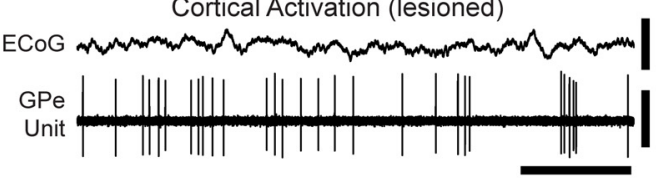

C

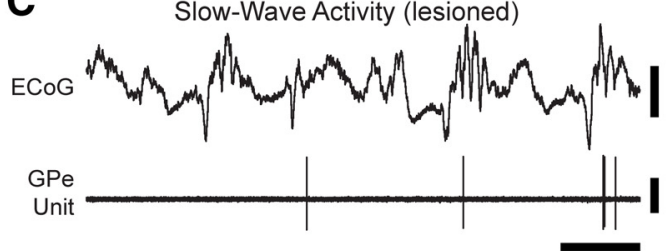

ECOG Cortical Activation (lesioned)

GPe
Unit

H

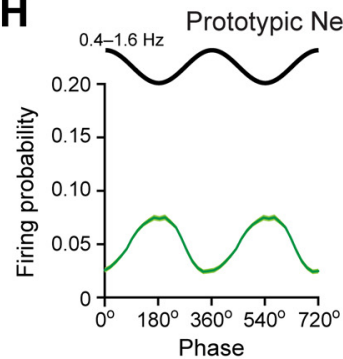

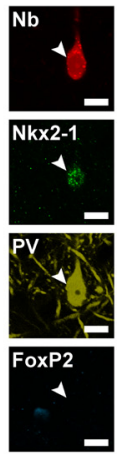

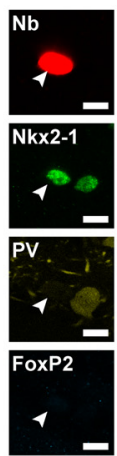

4

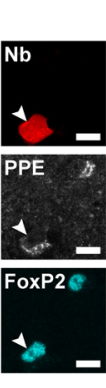

D

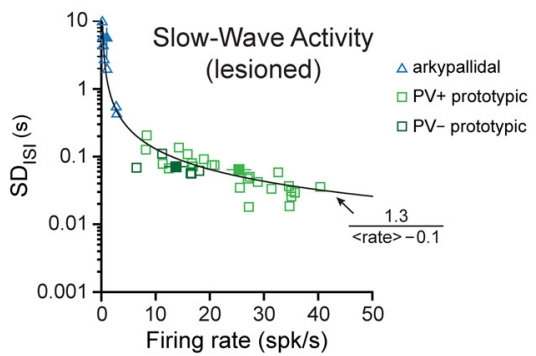

E

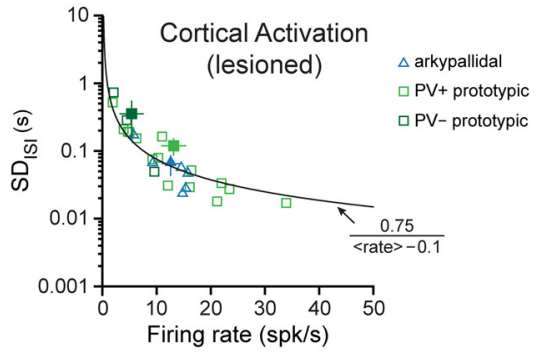

F

Prototypic Neurons (lesioned)
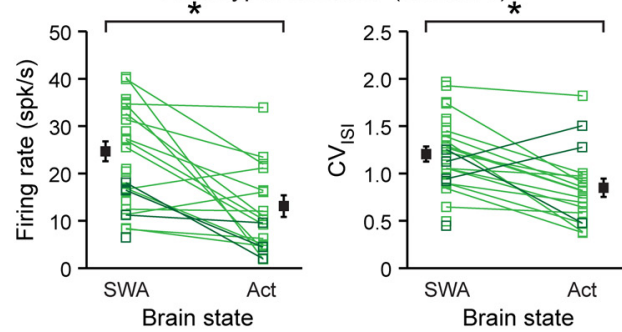

G Arkypallidal Neurons (lesioned)
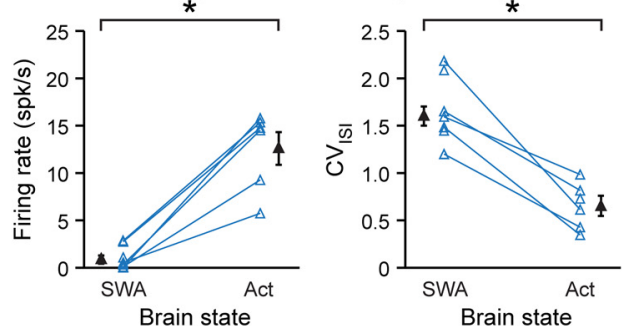

I

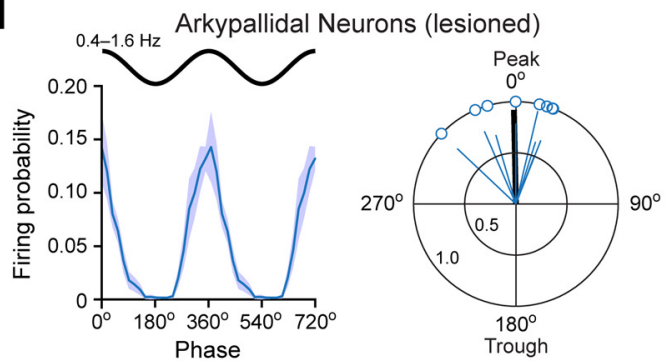

Figure 10. Molecularly identified prototypic neurons and arkypallidal neurons recorded in 6-OHDA-lesioned animals. $A$, Typical single-unit activity of a PV ${ }^{+}$prototypic GPe neuron recorded in a 6-OHDAlesioned adult rat. During SWA, the unit fired at relatively high rates and in time with cortical slow oscillations. However, during cortical activation, it fired at comparatively low rates. This neuron (arrowhead) coexpressed Nkx2-1 and PV, but not FoxP2, thus identifying it as prototypic. $\boldsymbol{B}$, Typical activity of a $\mathrm{PV}^{-}$prototypic GPe neuron. $\boldsymbol{C}$, Typical single-unit activity of an arkypallidal neuron recorded in a lesioned rat. This neuron expressed FoxP2 and PPE, thus identifying it as arkypallidal. $\boldsymbol{D}$, Plot of $\mathrm{SD}_{\mid \mathrm{IS|}}$ against mean firing rate for each GPe neuron recorded in lesioned rats during SWA. Arkypallidal neurons and prototypic neurons tended to distribute within distinct and restricted aspects of the population firing rate/pattern continuum. $\boldsymbol{E}$, Same as in $\boldsymbol{D}$ but for GPe neurons recorded during cortical activation. There is substantial overlap in the firing properties of arkypallidal and prototypic neurons during this brain state. $\boldsymbol{F}, \mathbf{G}$, Firing rates and $\mathrm{VV}_{\text {ISI }}$ for each prototypic neuron $(\boldsymbol{F})$ and for each arkypallidal neuron $(\boldsymbol{G})$ recorded during $S W A$ and/or activation (Act). $\boldsymbol{H}, \boldsymbol{I}$, Linear phase histograms (left) and circular plots (right) for all spikes of all prototypic neurons $(\boldsymbol{H})$ or of all arkypallidal neurons $(\boldsymbol{I})$ with firing that was significantly phase-locked to cortical slow oscillations. On average, the firing of prototypic neurons in lesioned rats was strongly phase-locked to the troughs of cortical slow oscillations, whereas the firing of arkypallidal neurons was strongly locked to oscillation peaks. Vertical calibration bars: $A-C, 0.5 \mathrm{mV}$ (ECOG), $1 \mathrm{mV}$ (units). Horizontal calibration bars: $A-C, 1$ s. Scale bars: $A-C$, insets, $10 \mu \mathrm{m} .{ }^{*} p<0.05$ (Mann-Whitney U test).

rons, their firing regularities were similar $(p=0.026$ and $p=$ 0.148 , respectively, Mann-Whitney tests) (Fig. $10 B, D, F)$. Arkypallidal neurons fired at significantly lower mean rates $(<3 \mathrm{spk} / \mathrm{s}$; $p<0.001$ ) than prototypic neurons during SWA (Fig. 10C, $D, G$ ).
During SWA, the average firing rate of prototypic neurons in lesioned rats was not different from that of neurons in dopamineintact rats ( $p=0.188$, Mann-Whitney test). However, chronic dopamine loss profoundly altered the firing patterns of proto- 
typic neurons. Indeed, on average, the firing regularity of prototypic neurons was significantly decreased (i.e., higher $\mathrm{CV}_{\text {ISI }}$ values than those of neurons in dopamine-intact rats; $p<0.001$; see Fig. $10 F$ ), which was likely the result of their firing being strongly timed with ongoing cortical slow oscillations (Fig. $10 A, B)$. In line with this, the firing of all prototypic neurons $(n=$ 30) was significantly phase-locked to cortical slow oscillations in lesioned rats (Rayleigh's Uniformity Tests); and in further contrast to the situation in dopamine-intact animals, all prototypic neurons in lesioned rats preferentially fired around the oscillation troughs (i.e., at phase angles between $>90^{\circ}$ and $<270^{\circ}$ ) (Fig. $10 A, B, H)$. The mean angle of the preferred phases of these prototypic neurons $\left(179.4 \pm 3.4^{\circ}\right)$ was significantly different from that of neurons in intact animals $(p<0.001$, Watson-Williams F-Test; Figs. $6 \mathrm{H}$ and $10 \mathrm{H}$ ). The vector lengths of prototypic neurons in lesioned animals were also significantly greater than those of neurons in dopamine-intact animals $(p<0.001$, Mann-Whitney test), further indicating that the preferred firing of individual neurons was comparatively more tightly locked after dopamine loss.

Chronic dopamine loss had relatively subtle effects on the firing of arkypallidal neurons. Compared with arkypallidal neurons recorded during SWA in dopamine-intact rats, those recorded in lesioned rats had similar average firing rates and regularities (Fig. 10C,G). Moreover, the spikes of all qualifying arkypallidal neurons $(n=8)$ in lesioned rats were again significantly locked to cortical slow oscillations, and all neurons preferentially fired around the oscillation peaks (Fig. 10C,I). The mean angle of the preferred phases of these arkypallidal neurons (358.8 $\pm 8.1^{\circ}$; Fig. $\left.10 I\right)$ was similar to that of arkypallidal neurons in intact animals (Fig. 6I). Notably, however, the preferred firing of individual neurons was more tightly locked in lesioned rats, as demonstrated by significantly longer vector lengths $(p=0.036)$. The differential influence of 6-OHDA lesions on the firing of the two GPe cell types during SWA did not arise because of any systematic difference in the power of coincident cortical slow oscillations.

Most prototypic neurons (18 of 30 ) were subsequently recorded during periods of spontaneous cortical activation (Fig. $10 A, B)$. In stark contrast to the scenario observed in dopamineintact rats (Fig. 6), the average firing rate of prototypic neurons in lesioned animals during cortical activation was significantly lower than that during SWA $(p<0.001)$, with almost all of the tested neurons ( 16 of 18 ) responding with decreases in their firing during transition from SWA to an activated brain state (Fig. $10 F)$. Furthermore, during cortical activation, prototypic neurons in lesioned rats were hypoactive compared with prototypic neurons in dopamine-intact rats (Figs. $6 F$ and $10 F ; p<0.001$ ). Most arkypallidal neurons (6 of 9) were also recorded during spontaneous cortical activation (Fig. 10C). The nature and magnitude of the average responses of arkypallidal neurons to brain state transitions in lesioned rats (Fig. 10G) were indistinguishable from those exhibited by arkypallidal neurons in dopamine-intact rats (Fig. 6G). Because arkypallidal neurons in lesioned rats robustly increased their firing during brain-state transition (Fig. $10 G$ ), whereas prototypic neurons decreased their firing (Fig. $10 F)$, a marked overlap in the firing properties of these two cell types emerged during cortical activation (Fig. 10E). Indeed, on average, the firing rates and regularities of arkypallidal neurons were not different from those of prototypic neurons in this brain state $(p=0.617$ and $p=0.271$, respectively, Mann-Whitney tests). Together, these data show: (1) chronic dopamine loss has a divergent impact on the firing of molecularly identified proto- typic GPe neurons and arkypallidal neurons in vivo; and (2) these two cell types can be readily distinguished in lesioned rats by their firing properties during SWA but not by their firing rate and regularities during cortical activation.

Finally, our in vitro and in vivo electrophysiological recordings of identified neurons provide further context for our use of molecular and structural data to define GABAergic GPe cells as either prototypic or arkypallidal neurons (Fig. 5). The complex molecular profiles of GPe neurons in particular call for a careful evaluation of the merits of this dichotomous division; for example, Nkx2-1 ${ }^{+} /$Lhx $6{ }^{+}$prototypic neurons could be further subdivided into those that additionally express PV/ER81 and those that do not (Fig. 5). Importantly, though, our analyses at the population level show that not only do $\mathrm{Nkx} 2-1^{+} / \mathrm{PV}^{+}$neurons and $\mathrm{Nkx} 2-1^{+} / \mathrm{PV}^{-}$neurons both innervate STN and striatum, but also that most of their electrophysiological properties are indistinguishable. Thus, the dichotomous grouping of GABAergic GPe neurons into prototypic and arkypallidal cell types is well supported by the current evidence and has clear conceptual utility.

\section{Discussion}

Here, we provide evidence of arkypallidal and prototypic neurons in the dopamine-intact GPe. We demonstrate that these two cell types are distinguished and defined by a host of specialized molecular, structural, and physiological properties. Our data converge to support the concept that a division of labor in GPe, as actuated by arkypallidal and prototypic neurons, extends beyond the disease state and is likely critical for the implementation of normal function.

\section{Molecular architecture of arkypallidal and prototypic neurons}

Our analyses afford new insights into the molecular architecture of prototypic and arkypallidal neurons, plus impartial definitions of their population sizes. Prototypic neurons are identified by coexpression of Nkx2-1 and Lhx6 (with many also expressing PV and ER81) and comprise approximately two-thirds of all GPe neurons. In contrast, arkypallidal neurons coexpress FoxP2 and Meis 2 and constitute just over one-fourth of all GPe neurons. The functional significance of this dichotomous cell classification is further emphasized by our findings that $\mathrm{Nkx} 2-1^{+} / \mathrm{Lhx} 6^{+}$prototypic neurons are the major GPe cell population innervating STN and that $\mathrm{FoxP}_{2}{ }^{+}$arkypallidal neurons do not innervate STN but rather innervate striatum. We observed that Lhx6 and PV are often coexpressed in prototypic neurons, but a recent study instead argues that mouse GPe contains two major cell populations that are distinguished by their expression of Lhx6 or PV (Mastro et al., 2014). This discrepancy might arise from species differences. However, this is unlikely because, in agreement with our results, other studies in mouse (Flandin et al., 2010; NóbregaPereira et al., 2010) show that ER81, PV, and Lhx6 are coexpressed in a major subset of GPe neurons.

Selective expression of transcription factor combinations unambiguously discriminates prototypic from arkypallidal neurons. Our definition of Nkx2-1, Lhx6, FoxP2, and Npas1 expression has particular relevance for understanding the ontogeny of GPe neurons, and our results help place reports of developmental heterogeneity (Flandin et al., 2010; Nóbrega-Pereira et al., 2010) in the context of functionally defined cell types in adult GPe. Indeed, because adult prototypic neurons coexpress Nkx21/Lhx6, this cell type almost certainly derives from the medial ganglionic eminence (MGE) of the embryonic subpallium (Sussel et al., 1999; Flames et al., 2007; Flandin et al., 2010; Nóbrega- 
Pereira et al., 2010). Importantly, work in mice suggests that approximately one-fourth of GPe cells are generated independent of Nkx2-1 expression/function and, thus, do not arise from the MGE; many of these "non-MGE" neurons are Npas1 ${ }^{+}$(Flandin et al., 2010; Nóbrega-Pereira et al., 2010). Because almost all arkypallidal neurons are $\mathrm{Npas}^{+}{ }^{+}$, we speculate that the subpallial domain(s) of origin of this cell type lie outside of MGE. However, a minority of prototypic neurons are also $\mathrm{Npas}^{+}$, in line with findings in juvenile mice that a subset of $\mathrm{Nkx2}-1^{+}$GPe neurons coexpress Npas1 (Flandin et al., 2010). It follows that Npas1 is not a definitive lineage tracer for GPe neurons and does not strictly delineate arkypallidal or prototypic neurons in adult rat. Nevertheless, Npas1 expression is highly selective for pallidostriatal neurons.

\section{Firing properties of GPe cell types in dopamine-intact brain} We provide the first working definitions of the in vivo firing properties of prototypic and arkypallidal neurons in the dopamine-intact brain. Across the whole population of recorded GPe neurons, firing rate and jitter were highly diverse but covaried almost continuously. Such a continuum of GPe neuron firing has been previously interpreted as evidence of a single cell type, albeit one with remarkably heterogeneous properties (Deister et al., 2013). Our recordings of identified neurons offer another perspective; each cell type discretely distributes at either end of this continuum and, on average, prototypic neurons fire at significantly higher rates and regularities than arkypallidal neurons. Thus, the distinct molecular and structural properties of prototypic and arkypallidal neurons are mirrored in their distinct firing properties. On the basis of firing rates/patterns during SWA, these two cell types can be distinguished with some confidence. However, there is more overlap in their firing properties during cortical activation. The activity profile of prototypic neurons is broadly similar to that of the majority of GPe units (of unknown cell type) recorded in awake dopamine-intact rats and monkeys; these units are typically classified as "high-frequency discharge" neurons that occasionally pause (DeLong, 1971; Anderson and Turner, 1991; Elias et al., 2007; Benhamou et al., 2012). Conversely, the firing rates/patterns of arkypallidal neurons are more akin to those of the $15 \%-25 \%$ of GPe units alternatively classified as "low-frequency discharge bursting" neurons (DeLong, 1971; Benhamou et al., 2012).

Our in vitro recordings of identified prototypic and arkypallidal neurons offer valuable insights into the intrinsic basis of the diverse firing observed in vivo. We determined that the autonomous firing of prototypic neurons was faster and had less jitter than that of arkypallidal neurons and that these differences cannot be readily explained by rate wandering (Deister et al., 2013). It is thus likely that the comparatively high firing rates and regularities of prototypic neurons in vivo partly arise from their ability to engage in more robust autonomous firing. The activity continuum that we and others observe in GPe in vitro (Deister et al., 2013) should be interpreted in light of prototypic and arkypallidal neurons exhibiting different autonomous firing properties. Taken with differences in other intrinsic properties (Table 1), our data lay foundations for defining the intrinsic physiological signatures of prototypic and arkypallidal neurons. Moreover, our finding of cell-type-dependent physiological diversity, together with the presumed sampling of both cell types in past in vitro studies, also helps explain why GPe neurons are reported to exhibit a wide array of intrinsic properties (Cooper and Stanford, 2000; Günay et al., 2008; Bugaysen et al., 2010; Chuhma et al., 2011; Miguelez et al., 2012).
Autonomous firing of GPe neurons depends on several membrane conductances, including $\mathrm{I}_{\mathrm{NaP}}$ and those mediated by $\mathrm{HCN}$, SK, $\mathrm{K}_{\mathrm{v}} 4$, and $\mathrm{Ca}_{\mathrm{vH}}$ channels (Chan et al., 2004; Mercer et al., 2007; Deister et al., 2009). Computer simulations suggest that distinct intrinsic properties similar to those of prototypic and arkypallidal neurons can be generated by varying the densities of these and other conductances (Günay et al., 2008). Notably, GPe neurons capturing a key feature of the arkypallidal phenotype (i.e., relatively low rates of autonomous firing) are predicted to have relatively low $\mathrm{I}_{\mathrm{NaP}}$ (Günay et al., 2008). Our finding that arkypallidal neurons have comparatively smaller $\mathrm{I}_{\mathrm{NaP}}$ offers one candidate mechanism for the diverse autonomous firing of the two cell types and agrees well with simulation predictions. Because arkypallidal neurons exhibited larger AHP magnitudes (during autonomous firing at least) and lower firing rates, one might also expect that they express higher densities of $\mathrm{Ca}_{\mathrm{vH}}$ and $\mathrm{K}_{\mathrm{v}} 4$ channels, respectively (Günay et al., 2008).

Firing of GPe neurons in vivo arises from a complex interplay between their intrinsic membrane properties and inputs, particularly those arising from striatum and STN. On average, the highfrequency firing of prototypic neurons was positively, but weakly, coupled to the peaks of cortical slow oscillations. Because GABAergic striatal projection neurons and glutamatergic STN neurons also fire most around these peaks (Magill et al., 2001; Sharott et al., 2012), the preferred firing of prototypic neurons better reflects periodic excitation from STN. However, the robust autonomous activity of prototypic neurons would ensure they continue to fire at high rates outside of rhythmic excitations. The low-frequency firing of arkypallidal neurons was strongly coupled to oscillation peaks, which again presumably results from rhythmic excitation by STN. However, their "weaker" autonomous activity, and inputs from prototypic neurons (Mallet et al., 2012), would ensure only modest firing outside of periodic excitations. Increased firing of prototypic and arkypallidal neurons upon transition to activated brain states probably arises because of increased STN output rather than altered striatal output (Magill et al., 2001; Mallet et al., 2005; Sharott et al., 2012).

\section{Impact of dopamine loss and wider implications}

Chronic dopamine loss markedly changed the activity of prototypic neurons, and their preferred firing phases became consistently aligned to slow oscillation troughs. Because both striatopallidal and STN neurons in 6-OHDA-lesioned rats are hyperactive, but continue to fire most during oscillation peaks (Mallet et al., 2006, 2008a; Zold et al., 2012), the altered firing phases of prototypic neurons likely arise because their input is now tipped in favor of inhibition from striatum. Abnormally efficacious inputs from striatum might also explain why prototypic neurons are hypoactive during cortical activation. After lesions, arkypallidal neurons continue to fire in time with oscillations peaks (albeit more tightly synchronized) and still increase their firing during activation. Therefore, firing of arkypallidal neurons is not as obviously constrained/shaped by striatum. This raises the possibility that prototypic and arkypallidal neurons are disparately innervated by striatum and/or STN. In support of this, computational modeling suggests that not only do prototypic neurons receive larger inputs from striatum, and smaller inputs from STN, compared with arkypallidal neurons, but also that these disparate connection strengths lead to the emergence of their "anti-phase" firing in parkinsonism (Nevado-Holgado et al., 2014). In conclusion, the divergent impact of dopamine loss on the activity of prototypic and arkypallidal neurons reiterates 
the importance of these dichotomous cell types for GPe function and dysfunction.

\section{References}

Agoston Z, Schulte D (2009) Meis2 competes with the Groucho co-repressor Tle4 for binding to Otx2 and specifies tectal fate without induction of a secondary midbrain-hindbrain boundary organizer. Development 136: 3311-3322. CrossRef Medline

Albin RL, Young AB, Penney JB (1989) The functional anatomy of basal ganglia disorders. Trends Neurosci 12:366-375. CrossRef Medline

Anderson ME, Turner RS (1991) A quantitative analysis of pallidal discharge during targeted reaching movement in the monkey. Exp Brain Res 86:623-632. Medline

Arber S, Ladle DR, Lin JH, Frank E, Jessell TM (2000) ETS gene Er81 controls the formation of functional connections between group Ia sensory afferents and motor neurons. Cell 101:485-498. CrossRef Medline

Baufreton J, Atherton JF, Surmeier DJ, Bevan MD (2005) Enhancement of excitatory synaptic integration by GABAergic inhibition in the subthalamic nucleus. J Neurosci 25:8505-8517. CrossRef Medline

Baufreton J, Kirkham E, Atherton JF, Menard A, Magill PJ, Bolam JP, Bevan MD (2009) Sparse but selective and potent synaptic transmission from the globus pallidus to the subthalamic nucleus. J Neurophysiol 102:532545. CrossRef Medline

Benhamou L, Bronfeld M, Bar-Gad I, Cohen D (2012) Globus pallidus external segment neuron classification in freely moving rats: a comparison to primates. PLoS One 7:e45421. CrossRef Medline

Berens P (2009) CircStat: a MATLAB toolbox for circular statistics. J Stat Soft 31:1-21.

Bevan MD, Booth PA, Eaton SA, Bolam JP (1998) Selective innervation of neostriatal interneurons by a subclass of neuron in the globus pallidus of the rat. J Neurosci 18:9438-9452. Medline

Biel M, Wahl-Schott C, Michalakis S, Zong X (2009) Hyperpolarizationactivated cation channels: from genes to function. Physiol Rev 89:847885. CrossRef Medline

Bugaysen J, Bronfeld M, Tischler H, Bar-Gad I, Korngreen A (2010) Electrophysiological characteristics of globus pallidus neurons. PLoS One 5:e12001. CrossRef Medline

Butt SJ, Sousa VH, Fuccillo MV, Hjerling-Leffler J, Miyoshi G, Kimura S, Fishell G (2008) The requirement of Nkx2-1 in the temporal specification of cortical interneuron subtypes. Neuron 59:722-732. CrossRef Medline

Campbell P, Reep RL, Stoll ML, Ophir AG, Phelps SM (2009) Conservation and diversity of Foxp2 expression in muroid rodents: functional implications. J Comp Neurol 512:84-100. CrossRef Medline

Chan CS, Shigemoto R, Mercer JN, Surmeier DJ (2004) HCN2 and HCN1 channels govern the regularity of autonomous pacemaking and synaptic resetting in globus pallidus neurons. J Neurosci 24:9921-9932. CrossRef Medline

Chuhma N, Tanaka KF, Hen R, Rayport S (2011) Functional connectome of the striatal medium spiny neuron. J Neurosci 31:1183-1192. CrossRef Medline

Cooper AJ, Stanford IM (2000) Electrophysiological and morphological characteristics of three subtypes of rat globus pallidus neurone in vitro. J Physiol 527:291-304. CrossRef Medline

Deister CA, Chan CS, Surmeier DJ, Wilson CJ (2009) Calcium-activated SK channels influence voltage-gated ion channels to determine the precision of firing in globus pallidus neurons. J Neurosci 29:8452-8461. CrossRef Medline

Deister CA, Dodla R, Barraza D, Kita H, Wilson CJ (2013) Firing rate and pattern heterogeneity in the globus pallidus arise from a single neuronal population. J Neurophysiol 109:497-506. CrossRef Medline

Delfs JM, Ellison GD, Mercugliano M, Chesselet MF (1995) Expression of glutamic acid decarboxylase mRNA in striatum and pallidum in an animal model of tardive dyskinesia. Exp Neurol 133:175-188. CrossRef Medline

DeLong MR (1971) Activity of pallidal neurons during movement. J Neurophysiol 34:414-427. Medline

DeLong MR (1990) Primate models of movement disorders of basal ganglia origin. Trends Neurosci 13:281-285. CrossRef Medline

Du T, Xu Q, Ocbina PJ, Anderson SA (2008) NKX2.1 specifies cortical interneuron fate by activating Lhx6. Development 135:1559-1567. CrossRef Medline
Elias S, Joshua M, Goldberg JA, Heimer G, Arkadir D, Morris G, Bergman H (2007) Statistical properties of pauses of the high-frequency discharge neurons in the external segment of the globus pallidus. J Neurosci 27: 2525-2538. CrossRef Medline

Erbel-Sieler C, Dudley C, Zhou Y, Wu X, Estill SJ, Han T, Diaz-Arrastia R, Brunskill EW, Potter SS, McKnight SL (2004) Behavioral and regulatory abnormalities in mice deficient in the NPAS1 and NPAS3 transcription factors. Proc Natl Acad Sci U S A 101:13648-13653. CrossRef Medline

Flames N, Pla R, Gelman DM, Rubenstein JL, Puelles L, Marín O (2007) Delineation of multiple subpallial progenitor domains by the combinatorial expression of transcriptional codes. J Neurosci 27:9682-9695. CrossRef Medline

Flandin P, Kimura S, Rubenstein JL (2010) The progenitor zone of the ventral medial ganglionic eminence requires $\mathrm{Nkx} 2-1$ to generate most of the globus pallidus but few neocortical interneurons. J Neurosci 30:28122823. CrossRef Medline

Glaser J, Greene G, Hendricks S (2007) Stereology for biological research with a focus on neuroscience. Williston, VT: MBF.

Günay C, Edgerton JR, Jaeger D (2008) Channel density distributions explain spiking variability in the globus pallidus: a combined physiology and computer simulation database approach. J Neurosci 28:7476-7491. CrossRef Medline

Hoover BR, Marshall JF (2002) Further characterization of preproenkephalin mRNA-containing cells in the rodent globus pallidus. Neuroscience 111:111-125. CrossRef Medline

Johnson DH (1996) Point process models of single-neuron discharges. J Comput Neurosci 3:275-299. CrossRef Medline

Kato S, Inoue K, Kobayashi K, Yasoshima Y, Miyachi S, Inoue S, Hanawa H, Shimada T, Takada M, Kobayashi K (2007) Efficient gene transfer via retrograde transport in rodent and primate brains using a human immunodeficiency virus type 1-based vector pseudotyped with rabies virus glycoprotein. Hum Gene Ther 18:1141-1151. CrossRef Medline

Kita H (2007) Globus pallidus external segment. Prog Brain Res 160:111133. CrossRef Medline

Kita H, Kita T (2001) Number, origins, and chemical types of rat pallidostriatal projection neurons. J Comp Neurol 437:438-448. CrossRef Medline

Kita H, Kitai ST (1991) Intracellular study of rat globus pallidus neurons: membrane properties and responses to neostriatal, subthalamic and nigral stimulation. Brain Res 564:296-305. CrossRef Medline

Kita H, Kitai ST (1994) The morphology of globus pallidus projection neurons in the rat: an intracellular staining study. Brain Res 636:308-319. CrossRef Medline

Lachaux JP, Rodriguez E, Martinerie J, Varela FJ (1999) Measuring phase synchrony in brain signals. Hum Brain Mapp 8:194-208. CrossRef Medline

Le Van Quyen M, Foucher J, Lachaux J, Rodriguez E, Lutz A, Martinerie J, Varela FJ (2001) Comparison of Hilbert transform and wavelet methods for the analysis of neuronal synchrony. J Neurosci Methods 111:83-98. CrossRef Medline

Lee T, Kaneko T, Taki K, Mizuno N (1997) Preprodynorphin-, preproenkephalin-, and preprotachykinin-expressing neurons in the rat neostriatum: an analysis by immunocytochemistry and retrograde tracing. J Comp Neurol 386:229-244. CrossRef Medline

Liodis P, Denaxa M, Grigoriou M, Akufo-Addo C, Yanagawa Y, Pachnis V (2007) Lhx6 activity is required for the normal migration and specification of cortical interneuron subtypes. J Neurosci 27:3078-3089. CrossRef Medline

Magill PJ, Bolam JP, Bevan MD (2001) Dopamine regulates the impact of the cerebral cortex on the subthalamic nucleus-globus pallidus network. Neuroscience 106:313-330. CrossRef Medline

Magill PJ, Pogosyan A, Sharott A, Csicsvari J, Bolam JP, Brown P (2006) Changes in functional connectivity within the rat striatopallidal axis during global brain activation in vivo. J Neurosci 26:6318-6329. CrossRef Medline

Magno L, Catanzariti V, Nitsch R, Krude H, Naumann T (2009) Ongoing expression of Nkx2.1 in the postnatal mouse forebrain: potential for understanding NKX2.1 haploinsufficiency in humans? Brain Res 1304:164186. CrossRef Medline

Magno L, Kretz O, Bert B, Ersözlu S, Vogt J, Fink H, Kimura S, Vogt A, Monyer H, Nitsch R, Naumann T (2011) The integrity of cholinergic 
basal forebrain neurons depends on expression of Nkx2-1. Eur J Neurosci 34:1767-1782. CrossRef Medline

Mallet N, Le Moine C, Charpier S, Gonon F (2005) Feedforward inhibition of projection neurons by fast-spiking GABA interneurons in the rat striatum in vivo. J Neurosci 25:3857-3869. CrossRef Medline

Mallet N, Ballion B, Le Moine C, Gonon F (2006) Cortical inputs and GABA interneurons imbalance projection neurons in the striatum of parkinsonian rats. J Neurosci 26:3875-3884. CrossRef Medline

Mallet N, Pogosyan A, Márton LF, Bolam JP, Brown P, Magill PJ (2008a) Parkinsonian beta oscillations in the external globus pallidus and their relationship with subthalamic nucleus activity. J Neurosci 28:1424514258. CrossRef Medline

Mallet N, Pogosyan A, Sharott A, Csicsvari J, Bolam JP, Brown P, Magill PJ (2008b) Disrupted dopamine transmission and the emergence of exaggerated beta oscillations in subthalamic nucleus and cerebral cortex. J Neurosci 28:4795-4806. CrossRef Medline

Mallet N, Micklem BR, Henny P, Brown MT, Williams C, Bolam JP, Nakamura KC, Magill PJ (2012) Dichotomous organization of the external globus pallidus. Neuron 74:1075-1086. CrossRef Medline

Marusich MF, Furneaux HM, Henion PD, Weston JA (1994) Hu neuronal proteins are expressed in proliferating neurogenic cells. J Neurobiol 25: 143-155. CrossRef Medline

Massi L, Lagler M, Hartwich K, Borhegyi Z, Somogyi P, Klausberger T (2012) Temporal dynamics of parvalbumin-expressing axo-axonic and basket cells in the rat medial prefrontal cortex in vivo. J Neurosci 32:1649616502. CrossRef Medline

Mastro KJ, Bouchard RS, Holt HA, Gittis AH (2014) Transgenic mouse lines subdivide external segment of the globus pallidus (GPe) neurons and reveal distinct GPe output pathways. J Neurosci 34:2087-2099. CrossRef Medline

Mercer JN, Chan CS, Tkatch T, Held J, Surmeier DJ (2007) Nav1.6 sodium channels are critical to pacemaking and fast spiking in globus pallidus neurons. J Neurosci 27:13552-13566. CrossRef Medline

Miguelez C, Morin S, Martinez A, Goillandeau M, Bezard E, Bioulac B, Baufreton J (2012) Altered pallido-pallidal synaptic transmission leads to aberrant firing of globus pallidus neurons in a rat model of Parkinson's disease. J Physiol 590:5861-5875. CrossRef Medline

Mink JW (1996) The basal ganglia: focused selection and inhibition of competing motor programs. Prog Neurobiol 50:381-425. CrossRef Medline

Nakamura KC, Sharott A, Magill PJ (2014) Temporal coupling with cortex distinguishes spontaneous neuronal activities in identified basal gangliarecipient and cerebellar-recipient zones of the motor thalamus. Cereb Cortex 24:81-97. CrossRef Medline

Nambu A, Llinaś R (1994) Electrophysiology of globus pallidus neurons in vitro. J Neurophysiol 72:1127-1139. Medline

Nambu A, Tokuno H, Takada M (2002) Functional significance of the cortico-subthalamo-pallidal 'hyperdirect' pathway. Neurosci Res 43:111117. CrossRef Medline

Nevado-Holgado AJ, Mallet N, Magill PJ, Bogacz R (2014) Effective connectivity of the subthalamic nucleus-globus pallidus network during Parkinsonian oscillations. J Physiol 592:1429-1455. CrossRef Medline

Nóbrega-Pereira S, Gelman D, Bartolini G, Pla R, Pierani A, Marín O (2010) Origin and molecular specification of globus pallidus neurons. J Neurosci 30:2824-2834. CrossRef Medline

Oren I, Nissen W, Kullmann DM, Somogyi P, Lamsa KP (2009) Role of ionotropic glutamate receptors in long-term potentiation in rat hippocampal CA1 oriens-lacunosum moleculare interneurons. J Neurosci 29:939-950. CrossRef Medline

Park YY, Johnston D, Gray R (2013) Slowly inactivating component of $\mathrm{Na}^{+}$ current in peri-somatic region of hippocampal CA1 pyramidal neurons. J Neurophysiol 109:1378-1390. CrossRef Medline

Pascale A, Gusev PA, Amadio M, Dottorini T, Govoni S, Alkon DL, Quattrone A (2004) Increase of the RNA-binding protein $\mathrm{HuD}$ and posttranscriptional up-regulation of the GAP-43 gene during spatial memory. Proc Natl Acad Sci U S A 101:1217-1222. CrossRef Medline
Paxinos G, Watson C (2007) The rat brain in stereotaxic coordinates, Ed 6 . Amsterdam: Academic (Elsevier).

Redgrave P, Prescott TJ, Gurney K (1999) The basal ganglia: a vertebrate solution to the selection problem? Neuroscience 89:1009-1023. CrossRef Medline

Reimers-Kipping S, Hevers W, Pääbo S, Enard W (2011) Humanized Foxp2 specifically affects cortico-basal ganglia circuits. Neuroscience 175:75-84. CrossRef Medline

Sadek AR, Magill PJ, Bolam JP (2007) A single-cell analysis of intrinsic connectivity in the rat globus pallidus. J Neurosci 27:6352-6362. CrossRef Medline

Sato F, Lavallée P, Lévesque M, Parent A (2000) Single-axon tracing study of neurons of the external segment of the globus pallidus in primate. J Comp Neurol 417:17-31. CrossRef Medline

Schwarting RK, Huston JP (1996a) Unilateral 6-hydroxydopamine lesions of meso-striatal dopamine neurons and their physiological sequelae. Prog Neurobiol 49:215-266. CrossRef Medline

Schwarting RK, Huston JP (1996b) The unilateral 6-hydroxydopamine lesion model in behavioral brain research: analysis of functional deficits, recovery and treatments. Prog Neurobiol 50:275-331. CrossRef Medline

Sharott A, Doig NM, Mallet N, Magill PJ (2012) Relationships between the firing of identified striatal interneurons and spontaneous and driven cortical activities in vivo. J Neurosci 32:13221-13236. CrossRef Medline

Siapas AG, Lubenov EV, Wilson MA (2005) Prefrontal phase locking to hippocampal theta oscillations. Neuron 46:141-151. CrossRef Medline

Smith Y, Parent A, Seguela P, Descarries L (1987) Distribution of GABAimmunoreactive neurons in the basal ganglia of the squirrel monkey (Saimiri sciureus). J Comp Neurol 259:50-64. CrossRef Medline

Smith Y, Bevan MD, Shink E, Bolam JP (1998) Microcircuitry of the direct and indirect pathways of the basal ganglia. Neuroscience 86:353-387. CrossRef Medline

Stacy RC, Demas J, Burgess RW, Sanes JR, Wong RO (2005) Disruption and recovery of patterned retinal activity in the absence of acetylcholine. J Neurosci 25:9347-9357. CrossRef Medline

Steriade M (2000) Corticothalamic resonance, states of vigilance and mentation. Neuroscience 101:243-276. CrossRef Medline

Surmeier DJ, Mercer JN, Chan CS (2005) Autonomous pacemakers in the basal ganglia: who needs excitatory synapses anyway? Curr Opin Neurobiol 15:312-318. CrossRef Medline

Sussel L, Marin O, Kimura S, Rubenstein JL (1999) Loss of Nkx2.1 homeobox gene function results in a ventral to dorsal molecular respecification within the basal telencephalon: evidence for a transformation of the pallidum into the striatum. Development 126:3359-3370. Medline

Swift GH, Liu Y, Rose SD, Bischof LJ, Steelman S, Buchberg AM, Wright CV, MacDonald RJ (1998) An endocrine-exocrine switch in the activity of the pancreatic homeodomain protein PDX1 through formation of a trimeric complex with PBX1b and MRG1 (MEIS2). Mol Cell Biol 18:5109_ 5120. Medline

von Engelhardt J, Eliava M, Meyer AH, Rozov A, Monyer H (2007) Functional characterization of intrinsic cholinergic interneurons in the cortex. J Neurosci 27:5633-5642. CrossRef Medline

Walters JR, Hu D, Itoga CA, Parr-Brownlie LC, Bergstrom DA (2007) Phase relationships support a role for coordinated activity in the indirect pathway in organizing slow oscillations in basal ganglia output after loss of dopamine. Neuroscience 144:762-776. CrossRef Medline

West MJ (1999) Stereological methods for estimating the total number of neurons and synapses: issues of precision and bias. Trends Neurosci 22: 51-61. CrossRef Medline

West MJ (2012) Basic stereology for biologists and neuroscientists. Cold Spring Harbor, NY: Cold Spring Harbor Laboratory.

Wichmann T, DeLong MR (1996) Functional and pathophysiological models of the basal ganglia. Curr Opin Neurobiol 6:751-758. CrossRef Medline

Zold CL, Escande MV, Pomata PE, Riquelme LA, Murer MG (2012) Striatal NMDA receptors gate cortico-pallidal synchronization in a rat model of Parkinson's disease. Neurobiol Dis 47:38-48. CrossRef Medline 